\title{
From Type II string theory towards BSM/dark sector physics
}

\author{
Gabriele Honecker \\ Institut für Physik (WA THEP), Johannes Gutenberg-Universität Mainz \\ D - 55099 Mainz, Germany \\ Gabriele.Honecker@uni-mainz.de
}

\begin{abstract}
Four-dimensional compactifications of string theory provide a controlled set of possible gauge representations accounting for BSM particles and dark sector components. In this review, constraints from perturbative Type II string compactifications in the geometric regime are discussed in detail and then compared to results from heterotic string compactifications and non-perturbative/non-geometric corners. As a prominent example, an open string realization of the QCD axion is presented. The status of deriving the associated low-energy effective action in four dimensions is discussed and open avenues of major phenomenological importance are highlighted. As examples, a mechanism of closed string moduli stabilization by D-brane backreaction as well as one-loop threshold corrections to the gauge couplings and balancing a low string scale $M_{\text {string }}$ with unisotropic compact dimensions are discussed together with implications on potential future new physics observations. For illustrative purposes, an explicit example of a globally consistent D6-brane model with MSSM-like spectrum on $T^{6} /\left(\mathbb{Z}_{2} \times \mathbb{Z}_{6} \times \Omega \mathcal{R}\right)$ is presented.
\end{abstract}

Keywords: String phenomenology \& cosmology; BSM physics; dark sector.

PACS numbers: 11.25.Wx, 11.25.Hf, 11.30.Fs, 12.60.-i, 14.70.Pw, 14.80.Va

\section{Contents}

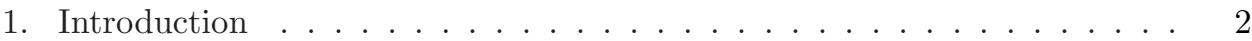

2. Geometric Compactifications of Perturbative Type II Strings and D-Branes 2

2.1. Consistency conditions . . . . . . . . . . . . . . . . . 3

2.2. Massless spectrum . . . . . . . . . . . . . . . . . 4

2.2.1. $U(1)$ factors and discrete $\mathbb{Z}_{n}$ symmetries . . . . . . . 5

2.2.2. Example: MSSM on $T^{6} /\left(\mathbb{Z}_{2} \times \mathbb{Z}_{6} \times \Omega \mathcal{R}\right) \ldots \ldots \ldots \ldots$

2.3. Towards the four-dimensional effective action . . . . . . . . . 9

2.3.1. Dimensional reduction of SUGRA, DBI and CS actions . . . 9

2.3.2. CFT \& beyond leading order . . . . . . . . . . . . . . 10

2.3.3. Moduli stabilization at the orbifold point . . . . . . . . 13

3. Heterotic String Theories and Non-Perturbative Regimes . . . . . . . 15

3.1. Heterotic $S O(32)$ on Calabi-Yau manifoldss . . . . . . . . . 16

3.2. Heterotic $E_{8} \times E_{8}$ and F-theory on Calabi-Yau manifolds . . . . . 17

3.3. Heterotic on Toroidal Orbifolds . . . . . . . . . . . . . . . . . . 19

3.4. Gepner/RCFT models . . . . . . . . . . . . . . . . . . . . . . . . . . 19

4. Open String Axions . . . . . . . . . . . . . . . . . . 20 
5. One-Loop Effects and Massive Gauge Bosons . . . . . . . . . . . . . . . . 21

6. Conclusions and Outlook . . . . . . . . . . . . . . . 23

\section{Introduction}

Even though string theory remains to date the arguably most successful framework for a unified description of Quantum Field Theory (QFT) and General Relativity - despite systematic extensive computer-aided searches such as in Refs. 1-18 - four dimensional string vacua with exactly the Standard Model or some GUT particle content as well as gauge and Yukawa couplings remain elusive. While the searches for chiral particle physics spectra are limited by the knowledge of (mostly) topological data for suitable six-dimensional compact backgrounds, in particular Calabi-Yau threefolds, deriving the low-energy effective action further necessitates a more detailed knowledge of the compact algebraic and differential geometry as well as techniques to quantize strings on such curved backgrounds.

One might thus argue that string theory is trapped between the juxtaposition of "anything goes" in terms of the assumed huge landscape of string vacua ${ }^{19,20}$ and "nothing goes" due to the lack of any explicitly known fully-fledged realistic string vacuum. As argued in this brief review, however, Beyond the Standard Model (BSM) and dark sector physics is considerably constrained within string theory compactifications as compared to purely field theoretical models, providing a guideline to search for new physics phenomena compatible with some UV completion. The focus here lies on representation theory and expanding the low-energy effective action in explicitly known corners of the string landscape and is thus complementary to landscape arguments such as in Ref. 21.

This review article is organized as follows: in section 2 generic model building rules from perturbative Type II superstring theories are presented with special emphasis on possibly allowed BSM/dark sector physics, which is compared in section 3 with model building rules from heterotic string theories and non-perturbative regimes. As two possible gateways to the dark sector, at first open string axions in particular as models for the QCD axion - are discussed in section 4, while oneloop gauge threshold corrections in Type II string theory and the relation to dark photons and Z' bosons for low values of $M_{\text {string }}$ are presented in section 5 . Section 6 contains the conclusions and outlook.

\section{Geometric Compactifications of Perturbative Type II Strings and D-Branes}

In this section, we briefly review the state-of-the-art in Type IIA orientifold model building with D6-branes, paying special attention to its limitations on new BSM and dark sector states. While the discussion focuses on the Type IIA case with D6-branes for the sake of the geometric intuition and of the clarity of the argument, it has been conjectured that generalizing the T-duality arguments from tori and toroidal orbifolds of Refs. 22,23 to mirror symmetry for Calabi-Yau threefolds leads 
to dual Type IIB orientifold models with either magnetized D9/D5- or D7/D3-brane systems, see e.g. Refs. 24, 25 for a comprehensive discussion and extended lists of references on perturbative Type IIB orientifold model building.

\subsection{Consistency conditions}

The string theoretic consistency conditions within the Type II string theory language can be grouped into two topological constraints and one using differential geometry:

(1) $\mathbf{R R}$ tadpole cancellation ensures that the RR charges among D-branes and O-planes cancel along compact directions. Since the O-plane charges are fixed by the choice of an (antiholomophic) involution $\mathcal{R}$ along the six compact dimensions accompanying (in Type IIA string theory) the worldsheet parity operation $\Omega$, the cycles $\Pi$ on which D-branes can wrap together with their multiplicity or 'stack size' $N$ are constrained. In terms of $\mathrm{D} 6_{a}$-branes and O6-planes wrapping compact three-cycles this is described by:

$$
\sum_{a} N_{a}\left(\Pi_{a}+\Pi_{a}^{\prime}\right)-4 \Pi_{O 6}=0,
$$

where $\Pi_{a}^{\prime} \equiv \mathcal{R}\left(\Pi_{a}\right)$ is the orientifold image of the three-cycle $\Pi_{a}$.

(2) The additional $\mathbb{Z}_{2}$-valued information carried by D-branes beyond their homology class $^{26}$ contained in the RR tadpole cancellation conditions of Eq. (1) is captured by the K-theory constraints, which are customarily formulated in terms of the absence of a field theoretical $S U(2) \simeq U S p(2)$ anomaly, ${ }^{27-29}$

$$
\Pi_{U S p(2)} \circ \sum_{a} N_{a} \Pi_{a}=0 \bmod 2 \quad \forall \text { probe } U S p(2),
$$

which is here for D6-branes written in terms of topological intersection numbers between three-cycles counting the number of chiral fermions in bifundamental representations. To verify these constraints in a given model, one first has to rewrite the symplectic basis of three-cycles in terms of orientifold-even, $\Pi_{i}^{+}$, and orientifold-odd, $\Pi_{i}^{-}$, ones $\left(i=0, \ldots, h^{21}\right)$ and then classify for which threecycles $\Pi_{c}^{+}=\sum_{i} X_{c}^{i} \Pi_{i}^{+}$(with wrapping numbers $X_{a}^{i} \in \mathbb{Z}$ ) D $6_{c^{-}}$-branes carry dimensionally enhanced gauge groups $U S p\left(2 N_{c}\right)$ or $S O\left(2 N_{c}\right)$.

(3) To ensure the stability of the string vacuum at the string scale $M_{\text {string }}$, supersymmetry (SUSY) is imposed. ${ }^{\mathrm{a}}$ In terms of D6-branes and O6-planes wrapped around three-cycles, the SUSY condition is equivalent to all wrapped threecycles being special Lagrangian (sLag) with the same calibration,

$$
\left.J_{1,1}^{\text {Kähler }}\right|_{\Pi_{a}}=0,\left.\quad \operatorname{Re}\left(\Omega_{3}\right)\right|_{\Pi_{a}}>0,\left.\quad \operatorname{Im}\left(\Omega_{3}\right)\right|_{\Pi_{a}}=0,
$$

${ }^{\text {a For }} M_{\text {string }}$ as low as $\mathcal{O}(\mathrm{TeV})$, one can argue that SUSY of the string vacuum is not mandatory but that e.g. Kaluza-Klein or winding modes should become visible in state-of-the-art collider experiments, e.g. at the LHC. ${ }^{30-41}$ 
where $J_{1,1}^{\text {Kähler }}$ denotes the Kähler form and $\Omega_{3}$ the holomorphic volume form of the compact Calabi-Yau threefold used for the compactification.

In practice, the three types of stringy consistency conditions are straightforwardly computed for factorisable torus backgrounds, $T^{6}=\left(T^{2}\right)^{3}$, and orbifolds thereof, $\left(T^{2}\right)^{3} / \Gamma$ (with $\Gamma=\mathbb{Z}_{N}$ or $\mathbb{Z}_{N} \times \mathbb{Z}_{M}$ with(out) discrete torsion), on which a full classification of three-cycles with typical Betti number $b_{3} \sim \mathcal{O}(10-50)$ is possible and for which Conformal Field Theory (CFT) methods can be invoked ${ }^{42-45}$ to not only cross-check the RR tadpole cancellation conditions in terms of vacuum amplitudes, but most importantly to determine the set of probe D-branes with $U S p(2)$ gauge groups for the K-theory constraints. ${ }^{16,28,46-48}$ Generalizing these results to so-called non-factorizable tori, e.g. $T^{6}=\left(T^{3}\right)^{2}$ or $T^{3} \times T^{1} \times T^{2}$, or orbifolds thereof is possible whenever the $s L a g$ cycles can be rewritten in terms of a factorized geometry, as was recently noticed ${ }^{49-51}$ when extending the first CFT computations ${ }^{52,53}$ to intersecting generic D6-branes with chiral spectra on non-factorizable $T^{6} /\left(\mathbb{Z}_{4} \times \Omega \mathcal{R}\right)$ backgrounds. For generic Calabi-Yau threefolds as compact backgrounds, already determining the overall sLag three-cycle $\Pi_{O 6}$ wrapped by the O6-planes in Eq. (1) is challenging ${ }^{54}$ with - to our best knowledge - no known strategy for classifying probe D-branes with $U S p(2)$ (and not $S O(2)$ ) gauge factors up to now.

\subsection{Massless spectrum}

Generic three-cycles $\Pi_{a}$ wrapped by stacks of $N_{a} \mathrm{D} 6_{a}$-branes provide $U\left(N_{a}\right)$ gauge factors, while orientifold-even three-cycles $\Pi_{c}^{+}$support either $U S p\left(2 N_{c}\right)$ or $S O\left(2 N_{c}\right)$ gauge groups of identical rank but enhanced dimension. The chiral spectrum can be straightforwardly computed from topological intersection numbers between threecycles as summarized in table 1, while the vector-like (massless and massive) spec-

\begin{tabular}{|c|c|}
\hline Representation & Multiplicity \\
\hline$\left(\mathbf{N}_{a}, \overline{\mathbf{N}}_{b}\right)$ & $\Pi_{a} \circ \Pi_{b}$ \\
\hline$\left(\mathbf{N}_{a}, \mathbf{N}_{b}\right)$ & $\Pi_{a} \circ \Pi_{b}^{\prime}$ \\
\hline$\left(\right.$ Anti $\left._{a}\right)$ & $\frac{\Pi_{a} \circ \Pi_{a}^{\prime}+\Pi_{a} \circ \Pi_{O 6}}{2}$ \\
\hline$\left(\mathbf{S y m}_{a}\right)$ & $\frac{\Pi_{a} \circ \Pi_{a}^{\prime}-\Pi_{a} \circ \Pi_{O 6}}{2}$ \\
\hline$\left(\mathbf{N}_{a}, \mathbf{2} \mathbf{M}_{c}\right)$ & $\Pi_{a} \circ \Pi_{c}$ \\
\hline
\end{tabular}

trum can (for D6-branes on three-cycles) to date only be accounted for by means of CFT computations on tori or toroidal orbifold backgrounds. There exist basically two complementary methods for counting all massless matter states:

(1) Any open string state can be explicitly constructed, if the compact background is sufficiently simple and the string quantization condition is known, and Chan- 
Paton labels can be associated to the different representations under the gauge groups, see e.g. Refs. 43-45 and 7,46 for the corresponding CFT computations with bulk and fractional D6-branes, respectively. Via this method, the chirality of each open string state and its localization along the compact directions is determined.

(2) The one-loop corrections to gauge couplings, including the full tower of massless and massive string excitations, can be computed by magnetically gauging string vacuum amplitudes and expanding in the gauging. ${ }^{55-57}$ The full amount of massless string states and the corresponding representations (without chirality assignments) can then be read off from the contributions of the different open string sectors to the beta function coefficients, which appear as prefactors of the $\frac{1}{\varepsilon}+\gamma_{E}-\ln 2 \simeq \ln \frac{M_{\mathrm{string}}^{2}}{\mu^{2}}$ terms in dimensional regularisation $\int d \ell \ell^{\varepsilon}[\ldots]$ of the corresponding vacuum amplitudes. ${ }^{47,58-60}$

The method of Chan-Paton labels is of vital importance when setting up the framework to compute Yukawa and higher $m$-point couplings, while the advantage of the one-loop gauge threshold computation is that (otherwise cumbersome) sign factors for distinguishing symmetric and antisymmetric representations are easily kept track of, and that extended computer scans and classifications of massless spectra in global D-brane models can be performed (with suitably powerful computers or clusters), when combining with the signs of intersection numbers to determine net-chiralities.

The first important message to take away at this point in view of BSM and/or dark sector physics is that any endpoint of an open string in perturbative Type II string theory transforms in the (anti)fundamental representation of the gauge group, which is supported on the (stack of) D-brane(s) the endpoint is confined to. Taking into account the orientifold projection, the following non-Abelian representations (plus their conjugates) under any $U\left(N_{a}\right) \times U\left(N_{b}\right) \times U S p\left(2 M_{c}\right) \times S O\left(2 M_{d}\right)$ group factor can appear:

$$
\begin{gathered}
\left(\mathbf{N}_{a}, \overline{\mathbf{N}}_{b}\right), \quad\left(\mathbf{N}_{a}, \mathbf{N}_{b}\right), \quad\left(\mathbf{N}_{x \in\{a, b,\}}, \mathbf{2 M}_{y \in\{c, d\}}\right), \quad\left(\mathbf{2 M}_{c}, \mathbf{2} \mathbf{M}_{d}\right), \\
\left(\mathbf{A d j}_{x \in\{a, b\}}\right), \quad\left(\mathbf{A n t i}_{x \in\{a, b, c, d\}}\right), \quad\left(\mathbf{S y m}_{x \in\{a, b, c, d\}}\right),
\end{gathered}
$$

while higher dimensional representations, such as e.g. $\left(\mathbf{A n t i}_{a}, \mathbf{N}_{b}\right)$ or $\left(\mathbf{N}_{a}, \mathbf{N}_{b}, \mathbf{N}_{c}\right)$, or spinorial representations, e.g. $\left(\mathbf{2}^{\mathbf{M}-\mathbf{1}_{ \pm}}\right)$, of $S O(2 M)$ gauge factors can never occur. $S O(10)$ Grand Unified Theories (GUTs) or exceptional gauge groups are thus not accessible from geometrically engineered perturbative Type II string vacua, while Pati-Salam, left-right symmetric models and MSSM-like spectra arise naturally.

\subsection{1. $U(1)$ factors and discrete $\mathbb{Z}_{n}$ symmetries}

The Abelian gauge factors within $\prod_{x} U\left(N_{x}\right)$ mix to form mass eigenstates, $U(1)_{X}=$ $\sum_{x} q_{x} U(1)_{x}$ with coefficients $q_{x} \in \mathbb{Q}$. In four spacetime dimensions, mass terms and 
couplings of $\operatorname{tr} F_{x}^{k, k \in\{1,2\}}$ to closed string two-forms $\mathcal{B}_{2}^{(i)}$ and their dual axions $\xi_{i}$ (with $d \mathcal{B}_{2}^{(i)}=m_{i} *_{4} d \xi_{i}$ for $m_{i} \in \mathbb{Z}$ depending on the three-cycle basis for a given sixdimensional compact space), which are in the Type IIA language complexifications of complex structure moduli and the dilaton, arise from the Chern-Simons action along the D6-branes, ${ }^{61}$

$$
\mathcal{S}_{C S} \supset \sum_{x} \sum_{i=0}^{h^{21}} \int_{\mathbb{R}^{1,3}}\left(Y_{x}^{i} \mathcal{B}_{2}^{(i)} \wedge \operatorname{tr} F_{x}+X_{x}^{i} \xi_{i} \operatorname{tr} F_{x} \wedge F_{x}\right),
$$

with three-cycle wrapping numbers $X_{x}^{i}, Y_{x}^{i} \in \mathbb{Z}$ stemming from the expansion $\Pi_{x}=$ $\sum_{i=0}^{3}\left[X_{x}^{i} \Pi_{i}^{+}+Y_{x}^{i} \Pi_{i}^{-}\right]$in orientifold-even and orientifold-odd parts introduced in section 2.1. A linear combination $U(1)_{X}$ remains massless and anomaly-free if the Stückelberg couplings to all two-forms in Eq. (5) vanishe, i.e. if $\sum_{x} q_{x} N_{x} Y_{x}^{i}=0$ for all $i$.

Classification of hypercharge embeddings: the origin of all matter representations from pairs of open string endpoints constrains the possible massless linear combinations which can reproduce the charge assignments of the hypercharge of all left- and right-handed quarks and leptons. Up to exchange of orientifold image D-branes $\left(x \leftrightarrow x^{\prime}\right.$ for $\left.x \in\{b, c, d\}\right)$, there exist only four different possibilities: ${ }^{62,63}$

$\left(q_{a}, q_{b}, q_{c}, q_{d}\right) \in\left\{\left(\frac{1}{6}, 0, \frac{1}{2}, \frac{1}{2}\right),\left(\frac{-1}{3}, \frac{-1}{2}, 0,0\right),\left(\frac{-1}{3}, \frac{-1}{2}, 0,1\right),\left(\frac{1}{6}, \frac{1}{2}, 0, \frac{-3}{2}\right)\right\}$,

to realize the Standard Model hypercharge and particle content on four (or three) stacks of D-branes with gauge groups $U(3)_{a} \times U(2)_{b} \times U(1)_{c}\left(\times U(1)_{d}\right)$, or for the 'standard embedding' $\left(\frac{1}{6}, 0, \frac{1}{2}, \frac{1}{2}\right)$ also $U(3)_{a} \times U S p(2)_{b} \times U(1)_{c}\left(\times U(1)_{d}\right)$.

In case of the 'standard embedding' and non-rigid D-branes, the 'right' symmetry $U(1)_{c}$ can arise from a breaking of a right-symmetric group $U S p(2)_{c}$ or $S O(2)_{c}$ along some flat direction in the Wilson line and displacement moduli space. Since in this case $U(1)_{c}$ remains massless, these models also possess a gauged baryon-lepton number $(B-L)$ symmetry with $\left(q_{a}, q_{b}, q_{c}, q_{d}\right)=\left(\frac{1}{3}, 0,0,1\right)^{2,7,60}$

$\mathbb{Z}_{n}$ symmetries: The original $U(1)_{x} \subset U\left(N_{x}\right)$ factors, or equivalently the massive linear combinations thereof, remain as perturbative global symmetries in the low-energy effective action. As expected in any quantum gravity framework, ${ }^{64,65}$ these continuous global symmetries are broken to discrete subgroups $\mathbb{Z}_{n}$ nonperturbatively, e.g. by D-brane instanton effects, provided that

$$
\sum_{x} k_{x} N_{x} Y_{x}^{i}=0 \bmod n \quad \forall i=0 \ldots h^{21},
$$

with integers $k_{x}$ such that $\operatorname{gcd}\left(n, k_{a}, k_{b}, \ldots\right)=1 .{ }^{48,63,66,67}$ These discrete symmetries provide the ultimate selection rules on couplings in the low-energy effective field theory. While the majority of examples to date consists of generation-independent $\mathbb{Z}_{n}$ symmetries, in Refs. 48,68 a generation-dependent $\mathbb{Z}_{2}$ was found, which can account for highly suppressed off-diagonal Yukawa couplings. 
Peccei-Quinn symmetries: In 1977, a spontenously broken global $U(1)_{P Q}$ symmetry was proposed to solve the strong CP problem. ${ }^{69}$ Within string theory models, it is natural to identify this $U(1)_{P Q}$ symmetry as one of the massive linear combinations (with mass $m_{U(1)_{P Q}} \sim M_{\text {string }}$ ) that are present as global symmetries at the perturbative level. Demanding that the QCD axion as well as the Higgs(es) and either right- or left-handed Standard Model particles are charged, boils down to two different possibilities for the 'standard embedding' of the hypercharge, namely $U(1)_{P Q}=U(1)_{b} \subset U(2)_{b}$ or $U(1)_{P Q}=U(1)_{c}-U(1)_{d}$ as discussed in more detail in section 4 . While non-perturbative effects will break such a $U(1)_{P Q}$ symmetry, some discrete subgroup can be preserved such as a $\mathbb{Z}_{3}$ gauge symmetry in the D6-brane model with MSSM spectrum ${ }^{18,70}$ displayed in section 2.2.2, see e.g. also Ref. 71 for a field theoretic model with $\mathbb{Z}_{3}$ symmetry or Ref. 72 in the context of F-theory.

\subsubsection{Example: $M S S M$ on $T^{6} /\left(\mathbb{Z}_{2} \times \mathbb{Z}_{6} \times \Omega \mathcal{R}\right)$}

To illustrate the above features, we present here an example of a globally consistent D6-brane model on the $T^{6} /\left(\mathbb{Z}_{2} \times \mathbb{Z}_{6} \times \Omega \mathcal{R}\right)$ orientifold with discrete torsion, ${ }^{18,70}$ which has Hodge numbers $\left(h^{11}, h^{21}\right)=\left(3_{\text {bulk }}+8_{\mathbb{Z}_{6}^{\prime}}+8_{\mathbb{Z}_{3}}, 1_{\text {bulk }}+2_{\mathbb{Z}_{6}}+2_{\mathbb{Z}_{3}}+(6+\right.$ $4+4)_{\mathbb{Z}_{2}}$ ) for the factorizable background lattice $\left(A_{1}\right)^{2} \times A_{2} \times A_{2}$. The a priori six different choices of orientations of the $\left(T^{2}\right)^{3}$ lattice under the antiholomorphic involution $\mathcal{R}$ can be reduced to two, ${ }^{16}$ i.e. $T_{(1)}^{2}$ is either of rectangular or of tilted shape, and due to the discrete torsion phase one O6-plane orbit $\Omega \mathcal{R}\left(\mathbb{Z}_{2}^{(i)}\right)$ has to be of exotic type, ${ }^{46,73}$ with two inequivalent choices, $\Omega \mathcal{R}$ or $\Omega \mathcal{R} \mathbb{Z}_{2}^{(3)}$, consistent with SUSY D6-branes on the so-called aAA-lattice orientation.

The $2 \times\left(h^{21}+1\right)_{\text {bulk }+\mathbb{Z}_{2}}=30$ three-cycles from the bulk and $\mathbb{Z}_{2}$-twisted sectors can be expanded in a symplectic basis of 15 orientifold-even and 15 orientifold-odd three-cycles as detailed in Ref. 18. For the choice of $\Omega \mathcal{R} \mathbb{Z}_{2}^{(3)}$ as the exotic O6plane orbit, SUSY solutions to the RR tadpole cancellation condition in Eq. (1) have the unusual feature that all D6-branes are parallel to the $\Omega \mathcal{R}\left(\mathbb{Z}_{2}^{(1)}\right)$-invariant planes along $T_{(1)}^{2}$ and at non-trivial angles along $T_{(1)}^{4} \equiv T_{(2)}^{2} \times T_{(3)}^{2}$. Under the orientifold projection, $h^{11}$ splits into $\left(h_{\mathbb{Z}_{6}^{\prime}}^{11}\right)^{+}=4$ and $\left(h_{\text {bulk }+\mathbb{Z}_{6}^{\prime}+\mathbb{Z}_{3}}\right)^{-}=15$, which count multiplets containing closed string vectors and Kähler moduli, respectively. The implications of all D6-branes wrapping the same one-cycle along $T_{(1)}^{2}$ on a potentially rather low value of the string scale $M_{\text {string }}$ in dependence of the radii $R_{1}^{(1)}, R_{2}^{(1)}$ for this specific kind of configuration will be further discussed in section 5 .

The SUSY D6-brane configuration for a specific MSSM-like particle spectrum, ${ }^{18}$ which satisfies all RR tadpole cancellation conditions of Eq. (1) and K-theory constraints of Eq. (2) is displayed in table 2. The 'standard embedding' of the hypercharge according to Eq. (6) constitutes the only massless Abelian gauge symmetry besides a $\mathbb{Z}_{3}$ symmetry that forms a remnant of the massive $U(1)_{P Q} \simeq U(1)_{c}-U(1)_{d}$ symmetry in this example. The massless open string spectrum charged under the low-energy gauge group $\left(S U(3)_{a} \times S U(2)_{b} \times S U(4)_{h}\right)_{\times U(1)_{Y}}^{\times \mathbb{Z}_{3}}$ is displayed in table 3 . 
8 Gabriele Honecker

Table 2. D6-brane configuration for a MSSM on $T^{6} /\left(\mathbb{Z}_{2} \times \mathbb{Z}_{6} \times \Omega \mathcal{R}\right)$.

\begin{tabular}{ccccccc}
\hline Brane & $\left(n_{i}, m_{i}\right)$ & $\frac{\text { angle }}{\pi}$ & $\mathbb{Z}_{2}^{(i)}$ & $(\vec{\tau})$ & $(\vec{\sigma})$ & group \\
\hline$a$ & $(1,0 ; 1,0 ; 1,0)$ & $(0,0,0)$ & $(+++)$ & $(0,1,1)$ & $(0,1,1)$ & $U(3)_{a}$ \\
$b$ & $(1,0 ;-1,2 ; 1,-2)$ & $\left(0, \frac{1}{2}, \frac{-1}{2}\right)$ & $(--+)$ & $(0,1,0)$ & $(0,1,0)$ & $U S p(2)_{b}$ \\
$c$ & $(1,0 ;-1,2 ; 1,-2)$ & $\left(0, \frac{1}{2}, \frac{-1}{2}\right)$ & $(-+-)$ & $(0,1,1)$ & $(0,1,1)$ & $U(1)_{c}$ \\
$d$ & $(1,0 ;-1,2 ; 1,-2)$ & $\left(0, \frac{1}{2}, \frac{-1}{2}\right)$ & $(+--)$ & $(0,0,1)$ & $(0,0,1)$ & $U(1)_{d}$ \\
$h$ & $(1,0 ; 1,0 ; 1,0)$ & $(0,0,0)$ & $(--+)$ & $(0,1,1)$ & $(0,1,1)$ & $U(4)_{h}$ \\
\hline
\end{tabular}

Since this model does not possess any gauged $(B-L)$ symmetry, the chiral mul-

Table 3. Massless spectrum of a MSSM example on $T^{6} /\left(\mathbb{Z}_{2} \times \mathbb{Z}_{6} \times \Omega \mathcal{R}\right)$.

\begin{tabular}{|c|c|c|c|c|c|}
\hline $\begin{array}{l}\text { sector } \\
\text { matter }\end{array}$ & $\begin{array}{c}a b \\
3(\mathbf{3}, \mathbf{2} ; \mathbf{1})_{1 / 6}^{0}\end{array}$ & $\begin{array}{c}a c \\
6(\overline{\mathbf{3}}, \mathbf{1} ; \mathbf{1})_{1 / 3}^{1}\end{array}$ & $\begin{array}{c}a d \\
3(\mathbf{3}, \mathbf{1} ; \mathbf{1})_{-1 / 3}^{1}\end{array}$ & $\begin{array}{c}a d^{\prime} \\
3(\overline{\mathbf{3}}, \mathbf{1} ; \mathbf{1})_{-2 / 3}^{1}\end{array}$ & $\begin{array}{c}b c \\
3(\mathbf{1}, \mathbf{2} ; \mathbf{1})_{1 / 2}^{1} \\
+3\left[(\mathbf{1}, \mathbf{2} ; \mathbf{1})_{1 / 2}^{1}+\text { h.c. }\right]\end{array}$ \\
\hline $\begin{array}{l}\text { sector } \\
\text { matter }\end{array}$ & $\begin{array}{c}b d \\
6(\mathbf{1}, \mathbf{2} ; \mathbf{1})_{-1 / 2}^{1} \\
+2\left[(\mathbf{1}, \mathbf{2} ; \mathbf{1})_{-1 / 2}^{1}+h . c .\right]\end{array}$ & $\begin{array}{c}c d \\
3(\mathbf{1}, \mathbf{1} ; \mathbf{1})_{0}^{1} \\
+3\left[(\mathbf{1}, \mathbf{1} ; \mathbf{1})_{0}^{1}+h . c .\right]\end{array}$ & $\begin{array}{c}c d^{\prime} \\
3(\mathbf{1}, \mathbf{1} ; \mathbf{1})_{1}^{0} \\
+3\left[(\mathbf{1}, \mathbf{1} ; \mathbf{1})_{1}^{0}+\text { h.c. }\right]\end{array}$ & & \\
\hline $\begin{array}{l}\text { sector } \\
\text { matter }\end{array}$ & $2\left[\left(\overline{\mathbf{3}}_{A}, \mathbf{1} ; \mathbf{1}\right)_{1 / 3}^{0}+\right.$ h.c. $]$ & $\begin{array}{c}b b+c c+d d \\
(5+4+5)(\mathbf{1}, \mathbf{1} ; \mathbf{1})_{0}^{0}\end{array}$ & $\begin{array}{c}d d^{\prime} \\
{\left[(\mathbf{1}, \mathbf{1} ; \mathbf{1})_{1}^{1}+\text { h.c. }\right]}\end{array}$ & $2\left[\begin{array}{c}a h \\
2\left[(\mathbf{3}, \mathbf{1} ; \overline{\mathbf{4}})_{1 / 6}^{0}+h . c .\right]\end{array}\right.$ & $\begin{array}{c}a h^{\prime} \\
{\left[(\mathbf{3}, \mathbf{1} ; \mathbf{4})_{1 / 6}^{0}+h . c .\right]}\end{array}$ \\
\hline $\begin{array}{l}\text { sector } \\
\text { matter }\end{array}$ & $\begin{array}{c}b h \\
3(\mathbf{1}, \mathbf{2} ; \mathbf{4})_{0}^{0}\end{array}$ & $\begin{array}{c}c h^{\prime} \\
6(\mathbf{1}, \mathbf{1} ; \overline{\mathbf{4}})_{-1 / 2}^{2}\end{array}$ & $\begin{array}{c}d h \\
3(\mathbf{1}, \mathbf{1} ; \overline{\mathbf{4}})_{1 / 2}^{2}\end{array}$ & $\begin{array}{c}d h^{\prime} \\
3(\mathbf{1}, \mathbf{1} ; \mathbf{4})_{1 / 2}^{2}\end{array}$ & $\begin{array}{c}h h^{\prime} \\
2\left[\left(\mathbf{1}, \mathbf{1} ; \mathbf{6}_{A}\right)_{0}^{0}+h . c .\right]\end{array}$ \\
\hline
\end{tabular}

tiplets in the $b c+b d$ sectors containing Higgs scalars and left-handed leptons can only be distinguished by their superpotential couplings to quarks and leptons as briefly discussed in section 2.3.2. Similarly, the $c d$ sector consists of chiral multiplets which contain not only three generations of right-handed Weyl neutrinos, but also candidates for the QCD axion as discussed further in section 4.

It is noteworthy that, even though the toroidal orbifold background contains singularities, the geometric engineering method provides exactly the same spectrum as expected in the smooth Calabi-Yau case, after neutral closed string blow-up and deformation modes have been used. This holds true even though some of the deformation moduli will be stabilized at the singular orbifold point via couplings to D6-branes as discussed further in section 2.3.3. This observation on the matter spectrum is in contrast to heterotic orbifolds and Gepner models discussed in section 3.3 and 3.4, respectively, where slight enhancements of gauge groups and representations as well as shifts of $U(1)$ charges in twisted sector can occur. In the case of heterotic orbifold models, geometric moduli can moreover not be uniquely distinguished from matter fields since all originate on equal footing from closed strings. 


\subsection{Towards the four-dimensional effective action}

While the chiral spectrum on intersecting D6-branes can be computed solely from topological intersection numbers of the corresponding three-cycles on any CalabiYau threefold, already the massless vector-like matter spectrum requires a more refined knowledge of the compact six-dimensional background. The situation becomes even more challenging when trying to reproduce the orders of magnitude of and hierarchies among Standard Model or some GUT gauge and Yukawa couplings.

The present techniques rely on combining dimensional reductions of the tendimensional supergravity (SUGRA) as well as Dirac-Born-Infeld (DBI) and ChernSimons (CS) actions along D-branes with CFT computations of scattering amplitudes for sufficiently simple toroidal (orbifold) backgrounds. The scope and stateof-the-art of these techniques will be discussed individually, and in section 2.3.3 complex structure moduli stabilization at the orbifold point will be discussed.

\subsubsection{Dimensional reduction of SUGRA, DBI and CS actions}

For any perturbative string theory, part of the tree-level effective action in four dimensions can be derived by dimensionally reducing the ten-dimensional SUGRA action. Expanding all massless closed string fields in terms of (cohomology classes of) differential forms along the compact space, or equivalently integrating the ten-dimensional fields over the dual cycles (or homology classes), yields the fourdimensional Kähler and complex structure moduli encoded in the Kähler form $J_{1,1}^{\mathrm{Kähler}}$ and holomorphic volume form $\Omega_{3}$, closed string vectors as well as the fourdimensional metric and the dilaton, along with their axionic and fermionic partner fields as worked out in detail in Ref. 74. Besides contributions to the moduli potential, the reduction of the Einstein-Hilbert term,

$$
\mathcal{S}_{\text {IIA }} \supset \frac{1}{2 \kappa_{10}} \int_{10 \mathrm{D}} \mathrm{d}^{10} x \sqrt{-g_{10}} e^{-2 \phi_{10}} \mathcal{R}_{10} \rightarrow \frac{1}{2 \kappa_{4}} \int_{\mathbb{R}^{1,3}} \mathrm{~d}^{4} x \sqrt{-g_{4}} \mathcal{R}_{4},
$$

provides an important relation among the characteristics of the string compactification and the four-dimensional Planck scale,

$$
\frac{M_{\text {Planck }}^{2}}{M_{\text {string }}^{2}}=\frac{4 \pi}{g_{\text {string }}^{2}} \frac{\operatorname{Vol}\left(C Y_{3}\right)}{\ell_{s}^{6}} .
$$

The string length is here defined in terms of the Regge slope $\alpha^{\prime}$ as $\ell_{s} \equiv 2 \pi \sqrt{\alpha^{\prime}}$, the string coupling as $g_{\text {string }} \equiv e^{\left\langle\phi_{10}\right\rangle}$ and the ten- and four-dimensional gravitational coupling constants as $\kappa_{10}^{2} \equiv \frac{\ell_{s}^{8}}{4 \pi}$ and $\kappa_{4}^{2} \equiv M_{\text {Planck }}^{-2}$, respectively.

In orientifolded Type II string theories, another part of the tree-level effective action in four dimensions can be obtained by dimensionally reducing the DBI and CS actions, see e.g. Refs. 75, 76 in the context of D6-branes,

$$
\mathcal{S}_{\mathrm{D} 6_{a}} \supset-\frac{1}{8 \pi \ell_{s}^{3}} \int_{7 \mathrm{D}} \mathrm{d}^{7} x e^{-\phi_{10}} F_{M N}^{a} F_{a}^{M N} \rightarrow-\frac{1}{4 g_{a, \text { tree }}^{2}} \int_{\mathbb{R}^{1,3}} \mathrm{~d}^{4} x F_{\mu \nu}^{a} F_{a}^{\mu \nu} .
$$


Generalizing this term from a single $\mathrm{D} 6_{a}$-brane carrying a $U(1)_{a}$ gauge group to non-Abelian gauge groups gives the relation

$$
\frac{4 \pi}{g_{a, \text { tree }}^{2}}=\frac{1}{4 c_{a} k_{a} g_{\text {string }}} \frac{\operatorname{Vol}\left(\Pi_{a}\right)}{\ell_{s}^{3}} \text { with } c_{a}=\left\{\begin{array}{ll}
1 & \text { bulk } \\
2 & \text { fractional } \\
4 & \text { rigid }
\end{array}, k_{a}=\left\{\begin{array}{ll}
1 & S U\left(N_{a}\right) \\
2 & U S p / S O\left(2 N_{a}\right)
\end{array},\right.\right.
$$

where the factor $c_{a}$ accounts for the fact that on toroidal orbifolds, the unimodular basis of three-cycles has the form $\Pi_{a}=\left(\Pi_{a}^{\mathrm{bulk}}+\Pi_{a}^{\mathbb{Z}_{2}}\right) / c_{a}$ with $\Pi_{a}^{\mathbb{Z}_{2}}$ a collection of exceptional three-cycles, which have zero volume at the singular orbifold point.

While the dimensional reduction is the easiest accessible technique using algebraic geometry on generic Calabi-Yau spaces, it is limited to tree-level effects stemming from massless string modes only. Moreover, the generalization of the full DBI action to stacks of D-branes and thus non-Abelian gauge groups is not known, and interactions originating from intersections of different stacks of D-branes, in particular Yukawa couplings, cannot be computed by this method.

For heterotic string theories, besides the tree-level supergravity action, the oneloop Green-Schwarz counter terms ${ }^{77}$ required for the cancellation of hexagonal gravitational, gauge and mixed anomalies in ten dimensions are known. These are matched by S-dual tree-level terms in the DBI and CS action within Type II string compactifications with D-branes. ${ }^{62,78,79}$ A brief comparison with heterotic model building, including also F-theory, is provided below in section 3 .

\subsubsection{CFT $\&$ beyond leading order}

Whenever the compactification background is simple enough and the string quantization on this space is explicitly known, CFT techniques can be employed to derive the one-loop corrections to the gauge couplings and the tree-level superpotential involving matter fields. Both types of contributions to the effective action are protected by perturbative non-renormalization theorems for supersymmetric field theories, but can receive non-perturbative contributions, e.g. from D-brane instantons, which can at least in principle also be computed by means of CFT. Also the fields, in particular the geometric moduli and the dilaton, entering the low-energy effective field theory might be subject to field redefinitions beyond leading order.

One-loop corrections to the gauge couplings: the tree-level and one-loop contributions to the gauge couplings can be computed in the same way as higher $m$ point couplings by inserting two vertex operators at the boundaries of open string worldsheets, which are disks at tree level and annuli and Möbius strips at one-loop level. The functional dependence on some closed string modulus is probed by adding the corresponding vertex operator in the bulk of the worldsheet. In Ref. 80, it was shown that (up to an undetermined numerical prefactor), the tree level result for the gauge coupling of a D6-brane agrees with Eq. (11) derived from dimensional reduction of the DBI action.

Fortunately, the one-loop corrections to the gauge couplings can also be com- 
puted in an alternative, somewhat simpler way without using vertex operators by instead magnetically gauging open string vacuum amplitudes ${ }^{55,56,81-83}$ with annulus and Möbius strip topologies along $\mathbb{R}^{1,3}$ and expanding in the gauging,

$$
b_{a} \ln \frac{M_{\text {string }}^{2}}{\mu^{2}}+\Delta_{a}=\sum_{b}\left[\mathcal{T}^{\mathcal{A}}\left(\mathrm{D} 6_{a}, \mathrm{D} 6_{b}\right)+\mathcal{T}^{\mathcal{A}}\left(\mathrm{D} 6_{a}, \mathrm{D} 6_{b^{\prime}}\right)\right]+\mathcal{T}^{\mathcal{M}}\left(\mathrm{D} 6_{a}, \mathcal{O} 6\right)
$$

where the usual identification $\frac{1}{\varepsilon}+\gamma_{E}-\ln 2 \simeq \ln \frac{M_{\text {string }}^{2}}{\mu^{2}}$ after dimensional regularization as anticipated in section 2.2 has been made. The results for intersecting bulk D6-branes on factorizable toroidal backgrounds or orbifolds thereof where first derived in Ref. 57,58 for vanishing vacuum expectation values of the continuous open string moduli. Partial results for rigid D6-branes were obtained in Ref. 59, and the formulas were systematically completed in Refs. 47,60 for bulk, fractional and rigid D6-branes. For rigid D6-branes, the contributions are summarized in the last column of table 4 with $V_{a b}^{(i)}=\frac{R_{1}^{(i)}}{R_{2}^{(i)}} n_{a}^{i} n_{b}^{i}+\frac{R_{2}^{(i)}}{R_{1}^{(i)}}\left(m_{a}^{i}+b_{i} n_{a}^{i}\right)\left(m_{b}^{i}+b_{i} n_{b}^{i}\right)$ for generic untilted $\left(b_{i}=0\right)$ or untilted $\left(b_{i}=\frac{1}{2}\right)$ tori or $V_{a b}^{(i)}=\frac{2 n_{a}^{i} n_{b}^{i}+n_{a}^{i} m_{b}^{i}+m_{a}^{i} n_{b}^{i}+2 m_{a}^{i} m_{b}^{i}}{\sqrt{3}}$ for hexagonal tori, $I_{a b}$ denoting toroidal intersection numbers and $I_{a b}^{\mathbb{Z}_{2}} \mathbb{Z}_{2}$-invariant intersection numbers dressed with sign factors due to $\mathbb{Z}_{2}$ eigenvalues and discrete Wilson lines $\tau_{i}$ as detailed in e.g. appendix A of Ref. 13. The lattice sums appearing in the gauge thresholds are defined ${ }^{47}$ as $\Lambda_{0,0}(v)=\ln \left(2 \pi v V \eta^{4}(i v)\right)$ and $\Lambda_{\tau, \sigma \neq 0,0}=\ln \left|e^{-\frac{\pi \sigma^{2} v}{4}} \frac{\vartheta_{1}\left(\frac{\tau-i \sigma v}{2}, i v\right)}{\eta(i v)}\right|^{2}$, with $\sigma_{i}^{a b} \equiv\left|\sigma_{i}^{a}-\sigma_{i}^{b}\right| \in\{0,1\}$ and $v_{i}$ the twotorus volumes in units of $\alpha^{\prime}$.

Table 4. Annulus contributions to beta function coefficients, Kähler metrics and 1-loop threshold corrections for rigid D6-branes.

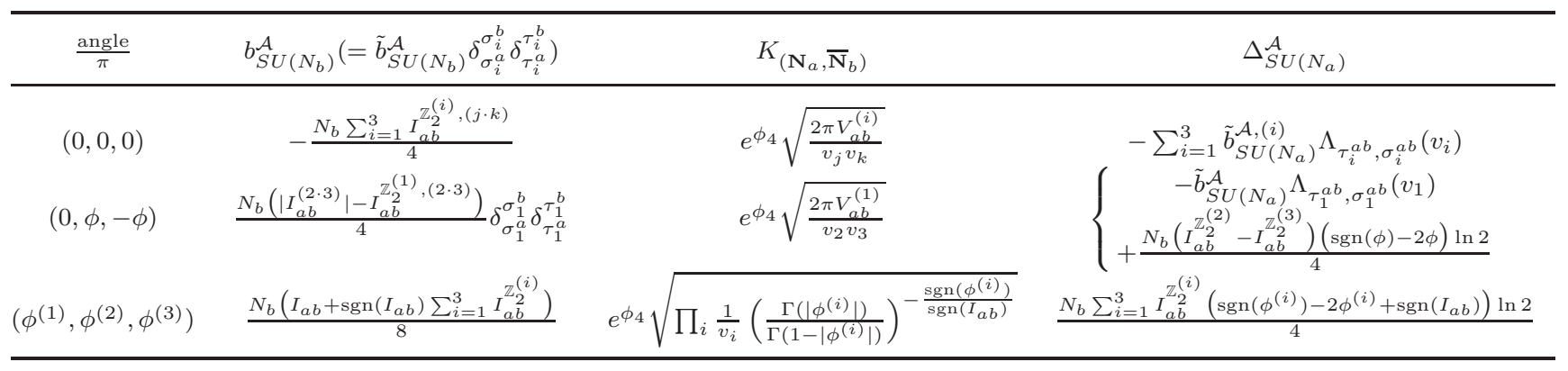

It is important to note here that all background configurations for the annulus topology of the worldsheet have been computed. For the Möbius strip topology, however, the beta function coefficients only match the field theory expectation, or more concretely the explicit construction of open string states and associated ChanPaton matrices, for D6-branes parallel along some two-torus $T_{(i)}^{2}$ if $b_{i} \sigma_{i} \tau_{i}=0$, i.e. either the torus is of rectangular shape or some of the open string moduli vacuum expectation values (vers, here displacements and Wilson lines $\sigma_{i}, \tau_{i} \in\{0,1\}$ for fractional and rigid D6-branes) vanish, as first noted in Ref. 46 and verified in 
the models constructed afterwards in Refs. 13,16,18. Since phenomenologically interesting models such as the MSSM example ${ }^{18}$ in table 2 (which has $b_{1}=0$, $b_{2}=b_{3}=1 / 2$ ) notoriously require $b_{i} \sigma_{i} \tau_{i} \neq 0$, it is of great importance to compute this missing piece of information on the one-loop gauge threshold. In section 5 , we will come back to this issue and discuss for which values of Kähler moduli the missing information can be neglected or will even cancel out among various contributions.

Finally, the one-loop gauge threshold can be decomposed into contributions to the holomorphic gauge kinetic function and leading-order expressions for the open string Kähler metrics by matching with the standard SUGRA expressions. ${ }^{47,59,84}$ At this point, it is particularly important for scalar potentials containing the shiftsymmetric terms ${ }^{85}\left(H_{u} \bar{H}_{d}+\right.$ h.c. $)$ that all tree level Kähler metrics on toroidal orbifold background of Type IIA string theory were found to be diagonal. It remains to be seen if this finding is specific to the simple backgrounds investigated so far, or if it generalizes to generic Calabi-Yau threefolds.

Yukawa and higher $m$-point functions: Each Yukawa coupling consists of a product of a (holomorphic) classical worldsheet instanton sum, which scales as $Y_{a b c} \propto e^{-\sum_{i} \text { Area }_{a b c}^{(i)}}$ in terms of the area bounded by three D6-branes $a, b, c$ with matter fields localised at the pairwise intersection points as computed in Refs. 86 for a factorisable six-torus $\prod_{i=1}^{3} T_{(i)}^{2}$, and a (non-holomorphic) quantum prefactor that was derived in Refs. 80, 87-89 again for the factorisable six-torus. These results are valid for bulk D6-branes (or fractions of pure bulk D6-branes on the $T^{6} /\left(\mathbb{Z}_{2} \times \mathbb{Z}_{2 M} \times \Omega \mathcal{R}\right)$ orientifolds without discrete torsion e.g. in Refs. 46, 90-94) with Yukawa couplings arising at non-vanishing angles on all three tori. ${ }^{\mathrm{b}}$ The phenomenologically appealing models with a reduced or vanishing number of open string moduli, however, require fractional $^{2,6,7,49,60,97-103}$ or rigid $^{13,16,18,46,70,73,104}$ D6-branes, respectively. In contrast to the simple torus models, chiral matter can here also arise at intersections with one vanishing angle, e.g. $(0, \phi,-\phi)$, since the $\mathbb{Z}_{2}$ symmetries break the naive $\mathcal{N}=2$ SUSY to $\mathcal{N}=1$ only. The argument that the CFT computation will give a vanishing result does thus not hold.

Alternatively, it is known $80,87,89,105,106$ that the quantum prefactor contains the Kähler metrics, $Y_{a b c} \propto\left(K_{a b} K_{b c} K_{c a}\right)^{-1 / 2} e^{\kappa_{4}^{2} \mathcal{K} / 2}$ with $\mathcal{K}$ the Kähler potential, of the relevant matter fields, which were determined to leading order as a byproduct of the gauge threshold computation in table 4 . If the different particle generations arise from intersections at different angles, this non-holomorphic prefactor can lead to an additional mild hierarchy ${ }^{101,102}$ beyond the exponentials of worldsheet areas.

In the MSSM example of section 2.2.2, the relative displacement $\sigma_{3}^{a b}=1$ between the D6-brane stacks $a$ and $b$ along $T_{(3)}^{2}$ in table 2 enforces a non-vanishing worldsheet area with the smallest suppression factors of trilinear Yukawa couplings $Y_{u} \propto e^{-\frac{4 v_{2}+v_{3}}{48}}$ and $e^{-\frac{v_{2}+4 v_{3}}{48}}$ involving the D6-brane stacks $a, b$ and $d^{\prime}$ and $Y_{d} \propto e^{-\frac{v_{3}}{48}}$ involving the stacks $a, b$ and $c$, since the $d_{R}$ particles stem from $a c$ sectors

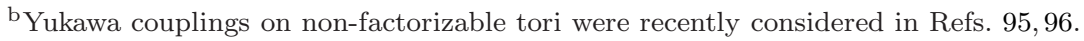


while the $u_{R}$ states arise from $a d^{\prime}$ sectors ${ }^{18}$ cf. table 3 . Since all ay sectors with $y \in\left\{b, c, c^{\prime}, d, d^{\prime}\right\}$ intersect at angles $\pi(0, \phi,-\phi)$ with $\phi= \pm \frac{1}{6}, \frac{1}{2}$, the quark Kähler metrics are universal, $K_{Q_{L}}=K_{u_{R}}=K_{d_{R}}=e^{\phi_{4}} \sqrt{\frac{R_{1}^{(1)}}{R_{2}^{(2)}} \frac{1}{v_{2} v_{3}}}=K_{H_{u}}=K_{H_{d}}$, and agree with the Kähler metrics of the Higgs fields arising also at angles $\pi(0, \phi,-\phi)$, but now with $\phi= \pm \frac{1}{3}$ in the $b c$ and $b d$ sectors.

Let us stress at this point that the full expression for Yukawa and higher $m$-point couplings at intersections of fractional or rigid D6-branes has to our best knowledge never been computed, and complex phases might arise when correctly defining the relevant boundary vertex operators. Such phases would be of major relevance to phenomenology. One possibility to check consistency of a first computation of this type would be to reproduce the one-loop gauge thresholds described above. This might also furnish the missing results for the Möbius strip topology with $b_{i} \sigma_{i} \tau_{i} \neq 0$.

Kähler metrics at one-loop and D-brane instantons: The above CFT results can be extended in two ways: at the perturbative level, in Refs. 107,108 the first computations of one-loop corrections to the Kähler metrics in IIA orientifold models were performed. The computations and resulting non-renormalization are, however, only valid for bulk D6-branes, due to the prefactors $\operatorname{tr} \gamma_{\mathbb{Z}_{2}}=0$ of the annulus amplitudes used there. All string vacua with chiral matter on fractional ${ }^{2,6,7,49,60,97-103}$ or rigid ${ }^{13,16,18,46,70,73,104,109}$ D6-branes violate this condition, making the extension of these computations to non-trivial twisted annulus contributions necessary. The second generalization of the above CFT methods consists in considering nonperturbative effects from D2-brane instantons wrapped along compact three-cycles. The computation of their contribution to the superpotential $\propto e^{-\mathcal{S}_{\text {inst }}}$ requires an integration over the D2-brane zero modes, which leads to a vanishing result unless the number of zero modes is minimal, see e.g. the review articles Refs. 110,111 for details. Non-perturbative corrections to the superpotential arise thus only from $O(1) \mathrm{D} 2$-branes wrapping rigid three-cycles. While these contributions have so far not been computed for the MSSM example of section 2.2.2, the classification of three-cycles wrapped by probe D6-branes with $U S p(2)$ gauge group in Ref. 18 is identical to the classification of $O(1)$ D2-branes, and the explicit form of the nonperturbative superpotential is thus in principle within reach.

In summary, a plethora of partial results on gauge and Yukawa couplings for D6-branes on toroidal orbifolds is known, but further intensive CFT computations are required to provide the exact formulas for gauge couplings and Kähler metrics beyond leading order, prefactors/phases of perturbative Yukawa couplings as well as non-perturbative contributions to the superpotential.

\subsubsection{Moduli stabilization at the orbifold point}

Generic Type IIA orientifold compactifications contain in the closed string sector $h_{-}^{11}$ Kähler and $h^{21}$ complex structure moduli, plus in the open string sector displacement and Wilson line moduli transforming in the adjoint representation of the 
gauge group. The latter kind of moduli is absent by construction if one uses rigid three-cycles. Toroidal orbifold backgrounds containing a $\mathbb{Z}_{2} \times \mathbb{Z}_{2}$ subgroup with non-trivial discrete torsion phase among the two $\mathbb{Z}_{2}$ factors provide such threecycles for $T^{6} /\left(\mathbb{Z}_{2} \times \mathbb{Z}_{2 M}\right)$ with $M \in\left\{1,3,3^{\prime}\right\},{ }^{13,16,18,46,73}$ as exemplified above in section 2.2.2. Additionally, in Ref. 112 it was conjectured that open string moduli receive a non-trivial potential via backreaction on the geometry, at least if closed string background fluxes are turned on, which, however, impedes model building with currently known geometric and CFT techniques. Also the $T^{6} /\left(\mathbb{Z}_{6}^{\prime} \times \Omega \mathcal{R}\right)$ model in Ref. 113 employs closed string background fluxes to stabilize geometric closed string moduli.

Since open and closed string sectors couple to each other, e.g. via the DBI and CS actions invoked in Eq. (10), it is natural to hypothesize that the presence of some D-brane - even without closed string fluxes - will stabilize (at least some of) the closed string moduli which it couples to. From a four-dimensional effective field theory point of view, the contribution to the scalar potential stems from a D-term, $V_{\text {scalar }}=\frac{1}{2} D_{a}^{2}+\ldots$, in which the Fayet-Iliopoulos (FI) term $\zeta_{i} \supset D_{a}$ corresponds to the vev of some geometric modulus. From a microscopic point of view, this hypothesis can be probed as follows: the volume of a fractional or rigid D6-brane at the singular orbifold point, where all twisted complex structure moduli have a vanishing vev, is given by the corresponding fraction of its bulk part. For SUSY D6-branes, the $s L a g$ condition in Eq. (3) implies that $\int_{\Pi_{a}} \Omega_{3} \stackrel{\text { SUSY }}{=} \operatorname{Vol}\left(\mathrm{D} 6_{a}\right)$, in other words the period is real. In Refs. 114-117 a hypersurface parameterization of the factorizable six-torus and its $\mathbb{Z}_{2}\left(\times \mathbb{Z}_{2}\right)$ orbifolds ${ }^{118}$ was implemented to allow for (complex structure) deformations away from the singular point. The sLag condition on the cycle $\Pi_{a}$ is then probed by computing the corresponding period and verifying if it remains real or develops an imaginary part upon deformation. By the relation in Eq. (11), any change in the cycle volume also changes the gauge coupling of the associated D-brane stack. The following different cases arise: ${ }^{114-117}$

(1) If a D6-brane couples via some orientifold-odd exceptional three-cycle within $\Pi_{a}^{\mathbb{Z}_{2}}$ to a twisted complex structure modulus, its deformation will break SUSY and generate a D-term potential. Deforming several singularities simultaneously leads to an additive scalar potential of the form $\propto \sum_{i} \zeta_{i}^{2}$.

(2) If a D6-brane only couples via some orientifold-even exceptional three-cycle to a twisted complex structure modulus, its deformation will change the value of the period, roughly speaking as $\pm \sqrt{\varepsilon}$ with $\varepsilon$ the SUSY deformation parameter and the sign factor depending on how the exceptional cycle enters $\Pi_{a}^{\mathbb{Z}_{2}}$. The gauge coupling of Eq. (11) thus experiences a flat direction.

(3) If a D6-brane does not couple directly to some twisted modulus, switching on a vev will only backreact on the D6-brane volume and gauge coupling via (gravitational) higher order effects. This statement holds for Kähler as well as complex structure moduli. 
In the MSSM example of table 2, the bulk complex structure modulus of $T_{(1)}^{2}$ and the three bulk Kähler moduli are not stabilized by their couplings to D-branes, and neither are the twelve twisted Kähler moduli at $\mathbb{Z}_{6}^{\prime}$ and $\mathbb{Z}_{3}$ singularities. In the $\mathbb{Z}_{2}^{(1)}$ twisted sector, three twisted complex structure moduli are not coupled to any D6-brane, two are stabilized by couplings to D6-branes $a, c, h$ and one provides a flat direction in the gauge couplings of stacks $b$ and $d$. While at the orbifold point, only relative $\mathbb{Z}_{2} \times \mathbb{Z}_{2}$ eigenvalues are relevant, upon deformation the absolute sign becomes important as anticipated in item (2). In any case, if the volume of brane $b$

shrinks, the volume of brane $d$ will increase, or vice versa. In the $\mathbb{Z}_{2}^{(2)}$ twisted sector, branes $c$ and $d$ couple to two of the four twisted deformation moduli, and in the $\mathbb{Z}_{2}^{(3)}$ twisted sector branes $a, d$ and $h$ do the same. Overall, we thus expect six twisted deformation moduli to be stabilized at the singular orbifold point by their couplings to the five stacks of D6-branes; one twisted deformation modulus constitutes a flat direction, which affects the $S U(2)_{W}$ and the $U(1)_{Y}$ gauge coupling strengths; finally the remaining deformation moduli possess flat directions, but backreact on the geometry via (gravitational) higher order effects. ${ }^{119}$

In principle, a FI term could be compensated by a suitable vev of some open string scalar. Giving a scalar partner of some MSSM fermion a vev would result in breaking the Standard Model gauge group, leaving only the option of giving a vev to the right-handed sneutrino in table 3 . But such a vev can at most compensate D-terms of either brane $c$ or $d$ due to the opposite $U(1)_{c} \times U(1)_{d}$ charges. Similarly, a vev of some right-handed squark would generically produce a D-term for stack $c$ or $d$ while eliminating that of stack $a$.

In summary, the mere existence of D-branes creates a backreaction on the geometry which leads to the stabilization of some geometric moduli, even before invoking closed string fluxes. The appeal of this mechanism lies in the fact that it avoids the no-go statement of simultaneously closed string fluxes and chiral fermions localized on some cycle. ${ }^{120,121}$ Of course, it might be attractive to switch on closed string fluxes and stabilize from the particle physics sector decoupled moduli, e.g. in view of viable inflationary potentials.

\section{Heterotic String Theories and Non-Perturbative Regimes}

In section 2, all allowed matter representations and corresponding gauge groups from geometrically engineered perturbative Type II string theory were discussed. Here, a complementary view on the allowed maximal rank of the overall gauge group by means of S-duality to the heterotic $S O(32)$ string theory will be discussed as well as enhancements of the allowed set of representations and simple Lie groups in the non-perturbative regime of Type II string theory, so-called F-theory, or the heterotic $E_{8} \times E_{8}$ string theory. Finally, we briefly discuss further enhancements due to singularities within the compact six-dimensional space. 


\subsection{Heterotic $S O(32)$ on Calabi-Yau manifoldss}

On non-singular Calabi-Yau threefolds, Type IIA orientifolds with intersecting D6branes have been conjectured to be mirror dual to Type IIB orientifolds with magnetized D7/D3- or D9/D5-brane systems, which in turn are conjectured to be S-dual to compactifications of the $S O(32)$ heterotic string theory. In the latter framework, the appearance of bifundamental representations can be seen from the embedding of $U\left(n_{i}\right)$ vector bundles $V_{i}$ within the ten-dimensional gauge group $S O(32)$, which leads to the breaking of the perturbative gauge group to $\prod_{i=1}^{K} U\left(N_{i} n_{i}\right) \times S O(2 M) \rightarrow$ $\prod_{i=1}^{K} U\left(N_{i}\right) \times U\left(n_{i}\right) \times S O(2 M)$ and all matter states arising from the decomposition of the adjoint representation of $S O(32):^{78,122,123}$

$$
496 \rightarrow\left(\begin{array}{c}
\left(\mathbf{A n t i}_{S O(2 M)}\right)+\sum_{i=1}^{K}\left(\mathbf{A d j}_{U\left(N_{i}\right)} ; \mathbf{A d j}_{U\left(n_{i}\right)}\right) \\
\sum_{i=1}^{K}\left[\left(\mathbf{A n t i}_{U\left(N_{i}\right)} ; \mathbf{S y m}_{U\left(n_{i}\right)}\right)+\left(\mathbf{S y m}_{U\left(N_{i}\right)} ; \mathbf{A n t i}_{U\left(n_{i}\right)}\right)+\text { h.c. }\right] \\
\sum_{i<j}\left[\left(\mathbf{N}_{i}, \mathbf{N}_{j} ; \mathbf{n}_{i}, \mathbf{n}_{j}\right)+\left(\mathbf{N}_{i}, \overline{\mathbf{N}}_{j} ; \mathbf{n}_{i}, \overline{\mathbf{n}}_{j}\right)+\text { h.c. }\right]+\sum_{i=1}^{K}\left[\left(\mathbf{2 M}, \mathbf{N}_{i}, \mathbf{n}_{i}\right)+\text { h.c. }\right]
\end{array}\right) .
$$

Non-perturbative five-branes in the $S O(32)$ heterotic string theory wrapped on compact two-cycles $\Gamma_{j}$ (Poincaré dual to the four-forms $\gamma_{j}$ ) support skyscaper sheaves and lead to the four-dimensional gauge group $U S p(2 M)$ with bifundamental matter arising with $U\left(N_{i}\right)$ gauge factors as well as further $U S p(2 M)$ 's. The massless matter spectrum is counted in terms of dimensions of cohomology and extension groups, and the net-chirality is determined by the corresponding Euler characteristic,

$$
\chi(W)=\int_{C Y_{3}}\left(\operatorname{ch}_{3}(W)+\frac{1}{12} c_{2}(T) c_{1}(W)\right) \quad \text { or } \quad-\int_{C Y_{3}} c_{1}\left(V_{i}\right) \wedge \gamma_{j},
$$

where $\operatorname{ch}_{n}(W)$ denotes the $n^{\text {th }}$ Chern character of the vector bundle $W, c_{1}(W)$ its first Chern class and $c_{2}(T)$ the second Chern class of the tangent bundle to the manifold. The chirality of the bifundamental representation $\left(\mathbf{N}_{i}, \overline{\mathbf{N}}_{j}\right)$ is for example computed using the bundle $W=V_{i} \otimes V_{j}^{*}$, while the second expression in Eq. (14) is valid for bifundamental matter arising from a vector bundle $V_{i}$ in combination with a five-brane wrapped on the two-cycle $\Gamma_{j}$.

The necessary string theoretic consistency conditions consist of the Bianchi identity on the three-form field strength, $d H_{3}=0$,

$$
\sum_{i} N_{i} \operatorname{ch}_{2}\left(V_{i}\right)-\sum_{j} M_{j} \gamma_{j}=-c_{2}(T) .
$$

The existence of well-defined spinors on the Calabi-Yau manifold - or in other words the absence of a global Witten anomaly - is guaranteed by the following 'mod 2' condition:

$$
\sum_{i} N_{i} c_{1}\left(V_{i}\right) \in H^{2}\left(C Y_{3}, 2 \mathbb{Z}\right)
$$

When reducing to rank $n_{i} \equiv 1$ for all $i$, one can see that via S-duality and mirror symmetry the Bianchi identity in Eq. (15) corresponds to the RR tadpole cancellation condition in Eq. (1), and the constraint on the existence of well-defined spinors in Eq. (16) is mapped to the K-theory constraint in Eq. (2). 
While in Type II string theories, the Green-Schwarz anomaly cancellation terms arise from the leading order of the DBI and CS actions, in compactifications of heterotic string theories, the counterterm arises at one-loop in the ten-dimensional supergravity action. ${ }^{77}$

The dual viewpoint of heterotic string theory provides an intuitive insight into the stringent upper bound on the rank of the total gauge group, since here the perturbative gauge group is in embedded into $S O(32)$ (or $E_{8} \times E_{8}$ as discussed in the next section 3.2), and any additional non-perturbative $U S p(2 M)$ gauge factor is constrained by the Bianchi identity in Eq. (15).

\subsection{Heterotic $E_{8} \times E_{8}$ and F-theory on Calabi-Yau manifolds}

The discussion of the Bianchi identity, well-definedness of spinors and net-chirality is completely analogous for the $S O(32)$ and $E_{8} \times E_{8}$ case. ${ }^{123,124}$ Also for compactifications of the $E_{8} \times E_{8}$ heterotic string, simple considerations of representation theory severely constrain any new physics state. Considering for example the series of decompositions $E_{8}^{(i)} \rightarrow E_{r_{i}} \times S U\left(n_{i}+m_{i}\right) \rightarrow E_{r_{i}} \times S U\left(n_{i}\right) \times S U\left(m_{i}\right) \times U(1)$ with $E_{r_{i}} \in\left\{E_{7}, E_{6}, S O(10), S U(5), S U(3) \times S U(2)\right\}$ and $r_{i}+n_{i}+m_{i}=9$ leads in the first step to the following decompositions of the adjoint representation of $E_{8}$ :

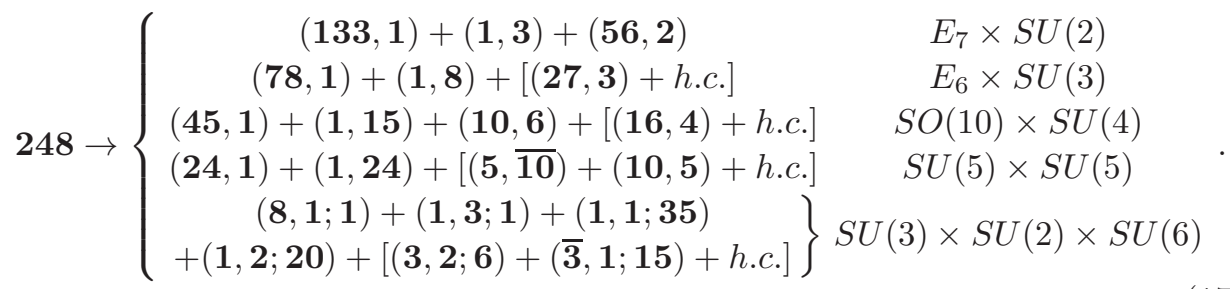

Besides the exceptional GUT group $E_{6}$, now also the spinor representation (16) of $S O(10)$ appears. It is also noteworthy that the only apparent representations of the $S U(n+m)$ factors in Eq. (17) are the fundamental $(\mathbf{n}+\mathbf{m})$, antisymmetrics $\left(\frac{(\mathbf{n}+\mathbf{m})(\mathbf{n}+\mathbf{m}-\mathbf{1})}{\mathbf{2}}\right)$ and $\left(\frac{(\mathbf{n}+\mathbf{m})(\mathbf{n}+\mathbf{m}-\mathbf{1})(\mathbf{n}+\mathbf{m}-\mathbf{2})}{\mathbf{6}}\right)$, and the adjoint $\left((\mathbf{n}+\mathbf{m})^{\mathbf{2}}-\mathbf{1}\right)$. This finding again poses constraints on the possible appearance of states with exotic charges. Let us for concreteness focus on the last case with $S U(n+m)=S U(6)$ and perform the second step of decomposing $S U(6) \rightarrow S U(n) \times S U(6-n) \times U(1)$ as exemplified in table 5. While one might naively try to embed only a $S U(n)$ bundle $V_{n}$

Table 5. Embedding of $S U(n) \times S U(6-n) \times U(1) \subset S U(6)$ bundles.

\begin{tabular}{|c|c||c|c|}
\hline$S U(6)$ rep. & $S U(5) \times U(1)$ & $S U(4) \times S U(2) \times U(1)$ & $S U(3) \times S U(3) \times U(1)$ \\
\hline$(\mathbf{6})$ & $(\mathbf{5})_{1}+(\mathbf{1})_{-5}$ & $(\mathbf{4}, \mathbf{1})_{1}+(\mathbf{1}, \mathbf{2})_{-2}$ & $(\mathbf{3}, \mathbf{1})_{1}+(\mathbf{1}, \mathbf{3})_{-1}$ \\
$(\mathbf{1 5})$ & $(\mathbf{1 0})_{2}+(\mathbf{5})_{-4}$ & $(\mathbf{6}, \mathbf{1})_{2}+(\mathbf{1}, \mathbf{1})_{-4}+(\mathbf{4}, \mathbf{2})_{-1}$ & $(\overline{\mathbf{3}}, \mathbf{1})_{2}+(\mathbf{1}, \overline{\mathbf{3}})_{-2}+(\mathbf{3}, \mathbf{3})_{0}$ \\
$(\mathbf{2 0})$ & $(\mathbf{1 0})_{-3}+(\overline{\mathbf{1 0}})_{3}$ & $(\mathbf{4}, \mathbf{1})_{-3}+(\overline{\mathbf{4}}, \mathbf{1})_{3}+(\mathbf{6}, \mathbf{2})_{0}$ & $(\mathbf{1}, \mathbf{1})_{-3}+(\mathbf{1}, \mathbf{1})_{3}+(\mathbf{3}, \overline{\mathbf{3}})_{-1}+(\overline{\mathbf{3}}, \mathbf{3})_{1}$ \\
$(\mathbf{3 5})$ & $(\mathbf{1})_{0}+(\mathbf{5})_{6}+(\overline{\mathbf{5}})_{-6}$ & $(\mathbf{1}, \mathbf{1})_{0}+(\mathbf{1 5}, \mathbf{1})_{0}+(\mathbf{1}, \mathbf{3})_{0}$ & $(\mathbf{1}, \mathbf{1})_{0}+(\mathbf{8}, \mathbf{1})_{0}+(\mathbf{1}, \mathbf{8})_{0}$ \\
& $+(\mathbf{2 4})_{0}$ & $+(\mathbf{4}, \mathbf{2})_{3}+(\overline{\mathbf{4}}, \mathbf{2})_{-3}$ & $+(\mathbf{3}, \overline{\mathbf{3}})_{2}+(\overline{\mathbf{3}}, \mathbf{3})_{-2}$ \\
\hline
\end{tabular}


and identify $U(1)_{Y} \simeq \frac{1}{6} U(1)$ in table 5 , this will generically lead to an enhancement of the four-dimensional gauge group. E.g. for $n=5$, both left-handed quarks $Q_{L}$ and right-handed up-type quarks $u_{R}$ are associated to the bundle $V_{5}$, while left-handed leptons $L$ and right-handed down-type quarks $d_{R}$ are linked to the bundle $\wedge^{2} V_{5}$, leading to an $S U(5)$ GUT instead of $S U(3) \times S U(2) \times U(1)_{Y}$. In Refs. 124-126, therefore a program to embed $U(n) \times U(m)$ bundles such that the hypercharge remains massless was initiated, with a plethora of systematic model searches using only line bundles conducted afterwards by various groups. ${ }^{11,12,14,127-129}$ The advantage of such line bundle constructions consists in the fact that line bundles are by definition stable.

While five-branes in compactifications of the $E_{8} \times E_{8}$ heterotic string theory contribute to the Bianchi identity analogously to Eq. (15), in contrast to the $S O(32)$ case each five-brane supports a tensor multiplet in six dimensions, which reduces to an Abelian gauge multiplet in four dimensions. Due to the very different origin of these $U(1)$ factors, matter fields remain uncharged.

The message to take away here is that again the origin of all charged states from the adjoint representation(s) of the ten-dimensional gauge group $E_{8}\left(\times E_{8}\right)$ severely constrains the representations under which any new physics particle and/or group might transform; for a comprehensive list on the relevant branchings of representations see e.g. the report in Ref. 130.

F-theory is defined as the strong coupling limit of Type IIB string theory with D7/D3-brane systems, where the string coupling is identified with the complex structure of an auxiliary two-torus or fibration. In this picture, Kodaira's ADEclassification of singularities (along the fiber) provides a geometric realisation of the non-Abelian gauge groups with sections corresponding to the Abelian factors, ${ }^{131,132}$ for an extended overview see e.g. the lecture notes in Ref. 133. The revival of four-dimensional F-theory models in $2008^{134,135}$ has consolidated the geometric brane engineering with the $E_{8} \times E_{8}$ heterotic dual (whenever existent) construction using vector bundles. Attempts of classifying all possible SCFTs in six dimensions have recently been made. ${ }^{136,137}$ While the question, how vast the four-dimensional landscape of string and F-theory vacua is, remains open, it is again obvious that new physics states are constrained by representation theory of non-Abelian gauge groups, which in the F-theory language corresponds to the classification of singularities.

Field theoretical investigations of both heterotic string theories on smooth manifolds are constrained by the limited scope of dimensionally reducing the tendimensional SUGRA action, just as for Type II orientifolds on smooth backgrounds. For perturbative Yukawa couplings in heterotic string theories, bundle cohomologies can be invoked to compute the holomorphic factors. ${ }^{138,139}$ Considerations on the existence of non-vanishing non-perturbative contributions to the superpotential can e.g. be found in Refs. 140-142 in relation to Gromov-Witten invariant of the underlying Calabi-Yau geometry. In the non-perturbative F-theory corner, in addition to the limitations on the effective action of the perturbative $E_{8} \times E_{8}$ heterotic string 
theory it remains to be clarified to what extent the perturbative SUGRA terms compete with e.g. non-perturvative instanton corrections.

\subsection{Heterotic on Toroidal Orbifolds}

In contrast to the toroidal orbifolds of Type II string theories discussed in section 2, in compactifications of heterotic string theory both geometric moduli and matter fields arise from excitations of closed strings. In the latter case, slight enhancements of representations and/or gauge groups at orbifold singularities occur. The characteristic features can already be observed in the overview of heterotic orbifold spectra in six dimensions in Ref. 143: the Bianchi identity and spinorial condition in Eqs. (15) and (16) translate into a quadratic and a linear condition on the shift vectors $\vec{V}$ and $\vec{v}$ in the gauge and torus lattice, respectively:

$$
N\left(\sum_{k} V_{k}^{2}-\sum_{i} v_{i}^{2}\right)=0 \bmod 2 \text { and } N \sum_{k} V_{k}=0 \bmod 2,
$$

for a perturbative $T^{2 n, n \in\{2,3\}} / \mathbb{Z}_{N}$ compactification. The total rank for any compactification without non-perturbative heterotic five-branes amounts to 16 , with either some additional massless Abelian gauge factor or a slightly enhanced nonAbelian gauge group. At orbifold singularities, twisted closed strings can combine into slightly enhanced representations, with in particular the spinor representation appearing in $S O(32)$ heterotic orbifold compactifications. If the gauge group contains $U(1)$ factors, charges in the $k^{\text {th }}$ twisted sector are typically shifted by $k / N$ compared to those expected from the decompositions in Eqs. (13) and (17).

The naive connection to heterotic compactifications on manifolds with vector bundles consists of identifying the shift vector with some line bundle, ${ }^{143,144}$

$$
\frac{1}{N}\left(1_{n_{1}}, 2_{n_{2}}, \ldots, 0_{n_{0}}\right) \rightarrow\left(L_{n_{1}}, L_{n_{2}}^{2}, \ldots, \mathcal{O}\right),
$$

but within four-dimensional models, matter representations at singularities often do not allow for a unique identification of the twisted geometric modulus, admitting instead for various resolutions to different Calabi-Yau manifolds. ${ }^{145-147}$

CFT methods to compute couplings for heterotic orbifold models were recently reconsidered ${ }^{148,149}$ due to the extended scans of landscape patches and studies of their phenomenological features. ${ }^{150,151}$

Free fermionic models have recently been shown to be dual ${ }^{152,153}$ to $T^{6} /\left(\mathbb{Z}_{2} \times \mathbb{Z}_{2}\right)$ orbifolds in the bosonic formulation as classified in Refs. 152,154.

\subsection{Gepner/RCFT models}

The classifications of particle spectra in Gepner or RCFT models ${ }^{1,3}$ belong morally to non-perturbative string theory corners away from the geometric engineering regime presented in section 2. Similarly to F-theory or heterotic orbifold models, enhancements of gauge groups occur. As a concrete example, hypercharge embeddings 
have been classified, ${ }^{155}$ adding the values $x=-\frac{1}{2}, 1, \frac{3}{2}$ in

$\left(q_{a}, q_{b}, q_{c}, q_{d}\right) \in\left\{\left(\frac{-1}{3}, \frac{-1}{2}, 0,0\right),\left(\frac{1}{6}, \frac{1}{2}, 0, \frac{-3}{2}\right)\right.$ and $\left(x-\frac{1}{3}, x-\frac{1}{2}, x, 1-x\right)$ with $\left.x=-\frac{1}{2}, 0, \frac{1}{2}, 1, \frac{3}{2}\right\}$

to the classification from a gauge quiver point of view in Eq. (6) of section 2.2.1. The extensive list of RCFT vacua thus again provides only a rather small number of possibilities to obtain the Standard Model gauge group.

\section{Open String Axions}

In 1977, the QCD axion was proposed as the Goldstone mode of a spontaneously broken global $U(1)_{P Q}$ symmetry. ${ }^{69,156}$ The natural embedding into Type II string theory consists in identifying the $U(1)_{P Q}$ symmetry with a massive gauge symmetry that acts as a global symmetry in perturbation theory, but is broken by non-perturbative effects such as D-brane instantons as discussed in section 2.2.1. Depending on the specific string vacuum, a discrete $\mathbb{Z}_{n}$ subgroup such as the $\mathbb{Z}_{3}$ gauge symmetry in the MSSM example of section 2.2.2 can remain as exact symmetry of the low-energy effective field theory. The QCD axion then arises as a massless open string state ${ }^{34,68,157-159}$ with $\mathbb{Z}_{n} \subset U(1)_{P Q}$ charge.

Since string theory vacua are customarily constructed to preserve $\mathcal{N}=1$ SUSY and thus have at least on pair of $\left(H_{u}, H_{d}\right)$ Higgs doublets, it is natural to extend the DFSZ axion model first proposed in $1981 .{ }^{160,161}$ In order to do so, the following set of constraints has to be met:

- The QCD axion $\sigma$ is charged under the massive $U(1)_{P Q}$ symmetry, but transforms as a singlet under the Standard Model gauge group.

- The Higgses $\left(H_{u}, H_{d}\right)$ also possess $U(1)_{P Q}$ charges in order to allow for a nontrivial Higgs-axion potential.

- To allow for all Yukawa couplings, either right- or left-handed quarks and leptons possess $U(1)_{P Q}$ charges.

While from a low-energy perspective, a shift by the hypercharge can be used to ensure that either left- or right-handed quarks (and similarly leptons) are neutral under $U(1)_{P Q}$, it is also useful to demand that the mass eigenstates of the massive $U(1)_{P Q}$ and massless $U(1)_{Y}$ symmetries are orthogonal in Type II string theory models. Based on these constraints, there exist only two possible realizations of $U(1)_{P Q}$ and the associated QCD axion for the 'standard' hypercharge embedding: 68,159

(1) $U(1)_{P Q} \simeq U(1)_{b} \subset U(2)_{b}$ : in this case, the QCD axion is realized as the antisymmetric representation (or its conjugate) of $U(2)_{b}$ and has thus the $U(1)_{P Q}$ charge \pm 2 . The left-handed Standard Model particles carry $U(1)_{P Q}$ charge, while the right-handed quarks and leptons are neutral. 
(2) $U(1)_{P Q} \simeq U(1)_{c}-U(1)_{d}$ : in this case, the $\mathrm{QCD}$ axion is the pseudoscalar partner of some right-handed neutrino transforming as $(\mathbf{1})_{ \pm 1, \mp 1}$ under $U(1)_{c} \times U(1)_{d}$ and thus again with charge \pm 2 under $U(1)_{P Q}$. The right-handed Standard Model particles carry $U(1)_{P Q}$ charge, whereas the left-handed ones remain neutral.

In both realizations, charge selection rules allow for the following Higgs-axion potential, ${ }^{159}$

$$
\begin{gathered}
V_{\mathrm{DFSZ}}^{\text {stringy }}= \\
=V_{D}+V_{F}+V_{\text {soft }} \\
\left.=H_{u}^{\dagger} H_{u}-v_{u}^{2}\right)^{2}+\lambda_{d}\left(H_{d}^{\dagger} H_{d}-v_{d}^{2}\right)^{2}+\lambda_{u d}\left|H_{u}^{\dagger} H_{d}\right|^{2}+\lambda_{\sigma}\left(\sigma^{\dagger} \sigma-v_{\sigma}^{2}\right)^{2} \\
+\left(\tilde{\lambda}_{u \sigma} H_{u}^{\dagger} H_{u}+\tilde{\lambda}_{d \sigma} H_{d}^{\dagger} H_{d}\right) \sigma^{\dagger} \sigma+\tilde{\lambda}_{u d}\left|H_{u} \cdot H_{d}\right|^{2} \\
+\widehat{\lambda}_{u d \sigma}\left(H_{u} \cdot H_{d} \sigma+\text { h.c. }\right)
\end{gathered}
$$

which differs from the original DFSZ model in the last line, $V_{\mathrm{DFSZ}}^{\text {original }} \supset H_{u} \cdot H_{d} \sigma^{2}$, due to the constraint that the complex scalar $\sigma$ obtains it $U(1)_{P Q}$ charge from the endpoints of some open string and thus has twice the charge of the field theoretically

predicted model. As a consequence the Higgs-axion coupling $\widehat{\lambda}_{u d \sigma}$ on the last line of Eq. (21) does not experience the $1 / M_{\text {Planck }}$ suppression of the original DFSZ model.

The soft breaking terms in Eq. (21) are expected to arise upon spontaneous SUSY breaking in a hidden sector, which is then via couplings to the gravity multiplet transmitted to the Higgs-axion sector. For example, in the MSSM-like model of section 2.2 .2 a gaugino condensate of the $S U(4)_{h}$ group might form if the associated vector-like matter states listed in table 3 decouple at a sufficiently high energy scale. On the other hand, mass terms for the vector-like down-type quarks arise via threepoint couplings to the axion multiplets $\Sigma$ with a somewhat weaker suppression of e.g. $e^{-\frac{v_{3}}{12}}$ than the Yukawa couplings discussed in section 2.3.2. Balancing all phenomenological constraints is therefore expected to require a considerable fine-tuning of Higgs and saxion veus.

\section{One-Loop Effects and Massive Gauge Bosons}

Balancing the string scale $M_{\text {string }}$, string coupling $g_{\text {string }}$ and Calabi-Yau and threecycle volumes $\operatorname{Vol}\left(C Y_{3}\right)$ and $\operatorname{Vol}\left(\Pi_{x}\right)$, respectively, such that gravity in four dimensions is weak, but the gauge couplings sufficiently strong, can in principle be achieved in two different ways:

(1) The tree-level gauge couplings in Eq. (11) can be rewritten as,

$$
\frac{4 \pi}{g_{a, \text { tree }}^{2}}=\frac{1}{2 \sqrt{2} c_{a} k_{a}} \frac{M_{\text {Planck }}}{M_{\text {string }}} \prod_{i=1}^{2} \sqrt{V_{a a}^{(i)}},
$$

with the square root of the left hand side of Eq. (9) appearing explicitly here. $V_{a a}^{(i)}$ has been defined in table 4 and only depends on toroidal wrapping numbers and complex structure moduli via $\left(R_{1}^{(i)} / R_{2}^{(i)}\right)^{ \pm 1}$. For either wrapping numbers 
$n_{a}^{i}=0$ or $m_{a}^{i}+b_{i} n_{a}^{i}=0$, a large hierarchy among the two radii can thus compensate a large ratio of $M_{\text {Planck }} / M_{\text {string }}$ in Eq. (22).

Type IIA orientifold compactifications on toroidal orbifolds usually require balancing RR charges among O6-planes extended along both $n_{a}^{i}=0$ and $m_{a}^{i}+b_{i} n_{a}^{i}=0$, which impedes large hierarchies among the associated radii and thus makes it natural to assum gauge coupling unification at $M_{\text {string }} \sim$ $M_{\mathrm{GUT}}=\mathcal{O}\left(10^{16}\right) \mathrm{GeV} \cdot{ }^{162}$

The MSSM example in table 2, however, has the special feature that $m_{x}^{1}=$ $0=b_{1}$ for all D6-brane stacks. On this particular orbifold background with discrete torsion, the O6-plane charge of the $\Omega \mathcal{R} \mathbb{Z}_{2}^{(2)}$-invariant plane is cancelled by the exotic charge of the $\Omega \mathcal{R} \mathbb{Z}_{2}^{(3)}$-invariant plane along $T_{(1)}^{2}$. A very unisotropic compactification with e.g. $R_{2}^{(1)} \sim 10^{7} R_{1}^{(1)}$ can thus compensate for a lower value of the string scale $M_{\text {string }} \sim 10^{12} \mathrm{GeV}$. The evaluation of the relevant length scales in the MSSM example of section 2.2.2 further gives the relation $1 / g_{S U(3)_{a}}^{2}=1 / g_{S U(4)_{h}}^{2}=2 / 3 g_{U S p(2)_{b}}^{2}=6 / 19 g_{U(1)_{Y}}^{2}$, i.e. the $S U(3)_{a}$ and $S U(4)_{h}$ gauge couplings at tree-level at $M_{\text {string }}$ are slightly stronger than those of the $U S p(2)_{b}$ and $U(1)_{Y}$ factors.

(2) The one-loop gauge threshold corrections from D6-branes at some vanishing angle displayed - for the annulus topology only - in table 4 contain lattice sums, which have the asymptotic behaviour $\Lambda_{\tau, \sigma}(v) \stackrel{v \rightarrow \infty}{\longrightarrow} \hat{c}_{\tau, \sigma} \frac{\pi v}{3}$ with $\hat{c}_{0,0}=-1$, $\hat{c}_{\tau, 1}=1$ and $\hat{c}_{1,0}=-2$. For highly unisotropic choices of two-torus volumes $v_{i} \sim v_{j} v_{j}$, the one-loop correction linear in the largest volume $v_{i}$ and with negative prefactor, i.e. relative displacement $\sigma^{i}=1$, can thus compensate the tree-level value $1 / g_{a, \text { tree }}^{2} \propto \sqrt{v_{1} v_{2} v_{3}}$.

In the MSSM example of section 2.2.2, the one-loop corrections of the QCD and hidden stack scale as $\delta_{\mathcal{A}}^{\text {loop }}\left(1 / g_{S U(3)_{a} / S U(4)_{h}}^{2}\right) \stackrel{v \rightarrow \infty}{\longrightarrow}$ const. $\times \frac{6 v_{1} \pm v_{2}}{6}$. The degeneracy of the two gauge couplings is thus lifted by the one-loop gauge threshold correction. The $S U(4)_{h}$ coupling becomes stronger, favouring the formation of a hidden sector gaugino condensate somewhere between $M_{\text {string }}$ und $M_{\text {weak }}$. At this point, it is noteworthy that including contributions from Möbius strip amplitudes leads to additional corrections $\delta_{\mathcal{M}}^{\text {loop }}\left(1 / g_{S U(3)_{a} / S U(4) h}^{2}\right) \propto$ $2 \Lambda_{0,0}\left(v_{1}\right)-\hat{\Lambda}_{1,1}\left(v_{2}\right)+\hat{\Lambda}_{1,1}\left(v_{3}\right)$ with unknown shape of the lattice sum $\hat{\Lambda}$ for tilted tori. Reliable field theoretical predictions are either possible in the regime $v_{1} \gg v_{2}, v_{3}$ or if $v_{2} \equiv v_{3}{ }^{119}$

In summary, the string scale can - even in simple toroidal orbifold set-ups - be chosen considerably below the GUT scale, opening up new arenas for new physics scenarios. The decay constant of e.g. a closed string axion participating in the Green-Schwarz mechanism scales as $f_{\xi} \sim M_{\text {string }}$, and can such an axion provide an explanation for dark matter if $M_{\text {string }}$ lies in an intermediary range of about $10^{9-12} \mathrm{GeV}$, which is also of interest to SUSY breaking. Going to the extreme case of $M_{\text {string }}=\mathcal{O}(\mathrm{TeV})$ on the other hand would allow for $Z^{\prime}$ bosons or dark photons that might become 
visible at the LHC or some future collider, see e.g.Refs. 34,163-168 for extended discussions.

\section{Conclusions and Outlook}

String theory as a framework for unifying General Relativity and QFT seemingly balances between "anything goes" and "nothing goes". Just as the Standard Model would be a very unlikely QFT if one were to take a statistical approach over all possible gauge groups and charged fermions, phenomenologically appealing embeddings of particle physics are very rare within the landscape of four-dimensional string vacua. The related representation theory is very well understood and provides a valuabe guideline for possible BSM and dark sector components compatible with some UV completion. Prominent examples are provided by open and closed string axions as well as massive gauge bosons. The role of the (pseudo)scalars can range from acting as inflaton, solving the strong $\mathrm{CP}$ problem, forming dark matter components or dark radiation, see e.g. the recent article in Ref. 169 for an extended list of references. Also massive gauge bosons can appear in various ways such as $Z^{\prime}$ bosons or dark photons, which mediate between the visible and a hidden sector via kinetic mixing ${ }^{170}$ with the hypercharge.

As argued here, the value of the string scale $M_{\text {string }}$ is strongly model-dependent within the framework of Type II orientifold compactifications. Highly unisotropic compactifications in combination with one-loop corrections to the gauge couplings can in principle lower $M_{\text {string }}$ to an intermediary range of about $10^{9-12} \mathrm{GeV}$, or sporadically even to the $\mathrm{TeV}$-range. In the latter case, an extreme amount of finetuning and stringy signatures at the LHC are to be expected.

While a classification in terms of gauge groups and matter representations seems to be within reach, deriving the four-dimensional effective action to a reliable level, where top-down scenarios of string-inspired field theoretical models of SUSY breaking, moduli stabilization or inflation can be tested, remains a major hurdle. As argued here, string phenomenology and string cosmology requires to carefully balance the beauty of geometrically engineering particle physics spectra on generic compact backgrounds versus the accessibility of the related (e.g. gauge and Yukawa) couplings using SUGRA or, at special lampposts of the string landscape, CFT methods. Concerted action in improving the knowledge of the low-energy effective action of string compactifications will ultimately pave the way to the understanding of UV-consistent new physics phenomena in four spacetime dimensions.

\section{Acknowledgments}

This work is partly supported by the Cluster of Excellence PRISMA DFG no. EXC 1098 and the DFG research grant HO 4166/2-2. 


\section{References}

1. T. Dijkstra, L. Huiszoon and A. Schellekens, Phys.Lett. B609, 408 (2005), arXiv:hep-th/0403196 [hep-th], doi:10.1016/j.physletb.2004.04.094.

2. G. and T. Ott, Phys.Rev. D70, 126010 (2004), arXiv:hep-th/0404055 [hep-th], doi: 10.1103/PhysRevD.70.126010,10.1103/PhysRevD.71.069902.

3. T. Dijkstra, L. Huiszoon and A. Schellekens, Nucl.Phys. B710, 3 (2005), arXiv:hep-th/0411129 [hep-th], doi:10.1016/j.nuclphysb.2004.12.032.

4. F. Gmeiner and M. Stein, Phys.Rev. D73, 126008 (2006), arXiv:hep-th/0603019 [hep-th], doi:10.1103/PhysRevD.73.126008.

5. F. Gmeiner, D. Lüst and M. Stein, JHEP 0705, 018 (2007), arXiv:hep-th/0703011 [hep-th], doi:10.1088/1126-6708/2007/05/018.

6. F. Gmeiner and G. Honecker, JHEP 0709, 128 (2007), arXiv:0708.2285 [hep-th], doi:10.1088/1126-6708/2007/09/128.

7. F. Gmeiner and G. Honecker, JHEP 0807, 052 (2008), arXiv:0806.3039 [hep-th], doi:10.1088/1126-6708/2008/07/052.

8. M. Robinson, G. Cleaver and M. B. Hunziker, Mod. Phys. Lett. A24, 2703 (2009), arXiv:0809.5094 [hep-th], doi:10.1142/S0217732309031843.

9. J. Rizos, Fortsch. Phys. 58, 758 (2010), arXiv:1003.0458 [hep-th], doi:10.1002/ prop. 201000047.

10. J. Rizos, Fortsch. Phys. 59, 1159 (2011), arXiv:1105.1243 [hep-ph], doi:10.1002/ prop. 201100057.

11. L. B. Anderson, J. Gray, A. Lukas and E. Palti, Phys.Rev. D84, 106005 (2011), arXiv:1106.4804 [hep-th], doi:10.1103/PhysRevD.84.106005.

12. L. B. Anderson, J. Gray, A. Lukas and E. Palti, JHEP 1206, 113 (2012), arXiv:1202.1757 [hep-th], doi:10.1007/JHEP06(2012)113.

13. G. Honecker, M. Ripka and W. Staessens, Nucl.Phys. B868, 156 (2013), arXiv:1209.3010 [hep-th], doi:10.1016/j.nuclphysb.2012.11.011.

14. L. B. Anderson, A. Constantin, J. Gray, A. Lukas and E. Palti, JHEP 1401, 047 (2014), arXiv:1307.4787 [hep-th], doi:10.1007/JHEP01(2014)047.

15. S. Groot Nibbelink and O. Loukas, JHEP 1312, 044 (2013), arXiv: 1308.5145 [hep-th], doi:10.1007/JHEP12(2013)044.

16. J. Ecker, G. Honecker and W. Staessens, Fortsch.Phys. 62, 981 (2014), arXiv:1409.1236 [hep-th], doi:10.1002/prop.201400066.

17. S. Groot Nibbelink, O. Loukas and F. Ruehle, Fortsch. Phys. 63, 609 (2015), arXiv:1507.07559 [hep-th], doi:10.1002/prop.201500041.

18. J. Ecker, G. Honecker and W. Staessens, Nucl. Phys. B901, 139 (2015), arXiv:1509.00048 [hep-th], doi:10.1016/j.nuclphysb.2015.10.009.

19. C. Vafa (2005), arXiv:hep-th/0509212 [hep-th]

20. A. N. Schellekens, Adv. Ser. Direct. High Energy Phys. 22, 155 (2015), doi:10.1142/ 9789814602686_0007.

21. K. R. Dienes and M. Lennek, Phys. Rev. D80, 106003 (2009), arXiv:0809.0036 [hep-th], doi:10.1103/PhysRevD.80.106003.

22. R.

Blumenhagen, B. Körs and D. Lüst, JHEP 0102, 030 (2001), arXiv:hep-th/0012156 [hep-th], doi:10.1088/1126-6708/2001/02/030.

23. C. Angelantonj and A. Sagnotti, Phys. Rept. 371, 1 (2002), arXiv:hep-th/0204089 [hep-th], doi:10.1016/S0370-1573(02) 00273-9,10.1016/S0370-1573(03)00006-1, [Erratum: Phys. Rept.376,no.6,407(2003)].

24. R. Blumenhagen, B. Körs, D. Lüst and S. Stieberger, Phys.Rept. 445, 1 (2007), 
arXiv:hep-th/0610327 [hep-th], doi:10.1016/j.physrep.2007.04.003.

25. L. E. Ibáñez and A. M. Uranga, Cambridge University Press (2012).

26. E. Witten, JHEP 9812, 019 (1998), arXiv:hep-th/9810188 [hep-th], doi:10.1088/ 1126-6708/1998/12/019.

27. E. Witten, Phys.Lett. B117, 324 (1982), doi:10.1016/0370-2693(82)90728-6.

28. A. M. Uranga, Nucl.Phys. B598, 225 (2001), arXiv:hep-th/0011048 [hep-th], doi: 10.1016/S0550-3213(00)00787-2.

29. F. G. Marchesano Buznego, Intersecting D-brane models, PhD thesis, Madrid, Autonoma U. (2003).

30. I. Antoniadis, N. Arkani-Hamed, S. Dimopoulos and G. Dvali, Phys.Lett. B436, 257 (1998), arXiv:hep-ph/9804398 [hep-ph], doi:10.1016/S0370-2693(98)00860-0.

31. G.

Shiu and S. H. H. Tye, Phys. Rev. D58, 106007 (1998), arXiv:hep-th/9805157 [hep-th], doi:10.1103/PhysRevD.58.106007.

32. E. Accomando, I. Antoniadis and K. Benakli, Nucl. Phys. B579, 3 (2000), arXiv:hep-ph/9912287 [hep-ph], doi:10.1016/S0550-3213(00)00123-1.

33. S. Cullen, M. Perelstein and M. E. Peskin, Phys. Rev. D62, 055012 (2000), arXiv:hep-ph/0001166 [hep-ph], doi:10.1103/PhysRevD.62.055012.

34. E. Kiritsis, Phys.Rept. 421, 105 (2005), arXiv:hep-th/0310001 [hep-th], doi:10. 1016/j.physrep.2005.09.001,10.1002/prop.200310120.

35. P. Burikham, T. Figy and T. Han, Phys. Rev. D71, 016005 (2005), arXiv:hep-ph/0411094 [hep-ph], doi:10.1103/PhysRevD.71. 016005,10.1103/PhysRevD.71.019905, [Erratum: Phys. Rev.D71,019905(2005)].

36. M. Chemtob, Phys. Rev. D78, 125020 (2008), arXiv:0808.1242 [hep-ph], doi:10. 1103/PhysRevD.78.125020.

37. L. A. Anchordoqui, H. Goldberg, D. Lüst, S. Nawata, S. Stieberger and T. R. Taylor, Nucl. Phys. B821, 181 (2009), arXiv:0904.3547 [hep-ph], doi:10.1016/j.nuclphysb. 2009.06.023.

38. M. Hashi and N. Kitazawa, JHEP 02, 050 (2012), arXiv:1110.3976 [hep-ph], doi: 10.1007/JHEP02(2012)050,10.1007/JHEP04(2012)011, [Erratum: JHEP04,011(2012)].

39. L. A. Anchordoqui, I. Antoniadis, D.-C. Dai, W.-Z. Feng, H. Goldberg, X. Huang, D. Lüst, D. Stojkovic and T. R. Taylor, Phys. Rev. D90, 066013 (2014), arXiv:1407.8120 [hep-ph], doi:10.1103/PhysRevD.90.066013.

40. P. Anastasopoulos and R. Richter, JHEP 12, 059 (2014), arXiv: 1408.4810 [hep-th], doi:10.1007/JHEP12(2014)059.

41. A. Celis, W.-Z. Feng and D. Lst, JHEP 02, 007 (2016), arXiv: 1512.02218 [hep-ph], doi:10.1007/JHEP02(2016)007.

42. M. Berkooz, M. R. Douglas and R. G. Leigh, Nucl. Phys. B480, 265 (1996), arXiv:hep-th/9606139 [hep-th], doi:10.1016/S0550-3213(96)00452-X.

43. R. Blumenhagen, L. Görlich and B. Körs, Nucl.Phys. B569, 209 (2000), arXiv:hep-th/9908130 [hep-th], doi:10.1016/S0550-3213(99)00795-6.

44. R. Blumenhagen, L. Görlich and B. Körs, JHEP 0001, 040 (2000), arXiv:hep-th/9912204 [hep-th], doi:10.1088/1126-6708/2000/01/040.

45. S. Förste, G. Honecker and R. Schreyer, Nucl.Phys. B593, 127 (2001), arXiv:hep-th/0008250 [hep-th], doi:10.1016/S0550-3213(00)00616-7.

46. S. Förste and G. Honecker, JHEP 1101, 091 (2011), arXiv:1010.6070 [hep-th], doi:10.1007/JHEP01(2011)091.

47. G. Honecker, Fortsch.Phys. 60, 243 (2012), arXiv:1109.3192 [hep-th], doi:10. 1002/prop.201100087. 
48. G. Honecker and W. Staessens, JHEP 10, 146 (2013), arXiv:1303.4415 [hep-th], doi:10.1007/JHEP10(2013)146.

49. A. Seifert and G. Honecker, Fortsch. Phys. 64, 416 (2016), arXiv:1511.03075 [hep-th], doi:10.1002/prop.201500071.

50. M. Berasaluce-Gonzlez, G. Honecker and A. Seifert, JHEP 08, 062 (2016), arXiv:1606.04926 [hep-th], doi:10.1007/JHEP08(2016)062.

51. M. Berasaluce-González, G. Honecker and A. Seifert (2016).

52. R. Blumenhagen, J. P. Conlon and K. Suruliz, JHEP 07, 022 (2004), arXiv:hep-th/0404254 [hep-th], doi:10.1088/1126-6708/2004/07/022.

53. S.

C. Timirgaziu and I. Zavala, JHEP 10, 025 (2007), arXiv:0707.0747 [hep-th], doi:10.1088/1126-6708/2007/10/025.

54. E. Palti, JHEP 0904, 099 (2009), arXiv:0902.3546 [hep-th], doi:10.1088/ 1126-6708/2009/04/099.

55. A. Abouelsaood, C. G. Callan, Jr., C. R. Nappi and S. A. Yost, Nucl. Phys. B280, 599 (1987), doi:10.1016/0550-3213(87)90164-7.

56. C. Bachas and C. Fabre, Nucl. Phys. B476, 418 (1996), arXiv:hep-th/9605028 [hep-th], doi:10.1016/0550-3213(96)00332-X.

57. D. Lüst and S. Stieberger, Fortsch.Phys. 55, 427 (2007), arXiv:hep-th/0302221 [hep-th], doi:10.1002/prop.200310335.

58. N. Akerblom, R. Blumenhagen, D. Lüst and M. Schmidt-Sommerfeld, Phys.Lett. B652, 53 (2007), arXiv:0705.2150 [hep-th], doi:10.1016/j.physletb.2007.06.060.

59. R. Blumenhagen and M. Schmidt-Sommerfeld, JHEP 0712, 072 (2007), arXiv:0711.0866 [hep-th], doi:10.1088/1126-6708/2007/12/072.

60. F.

and G. Honecker, Nucl.Phys. B829, 225 (2010), arXiv:0910.0843 [hep-th], doi: 10.1016/j.nuclphysb.2009.12.011.

61. L. E. Ibáñez, F. Marchesano and R. Rabadan, JHEP 0111, 002 (2001), arXiv:hep-th/0105155 [hep-th].

62. F. Gmeiner, R. Blumenhagen, G. Honecker, D. Lüst and T. Weigand, JHEP 0601, 004 (2006), arXiv:hep-th/0510170 [hep-th], doi:10.1088/1126-6708/2006/01/004.

63. P. Anastasopoulos, M. Cvetič, R. Richter and P. K. Vaudrevange, JHEP 1303, 011 (2013), arXiv:1211.1017 [hep-th], doi:10.1007/JHEP03(2013)011.

64. R. Kallosh, A. D. Linde, D. A. Linde and L. Susskind, Phys.Rev. D52, 912 (1995), arXiv:hep-th/9502069 [hep-th], doi:10.1103/PhysRevD.52.912.

65. T. Banks and N. Seiberg, Phys.Rev. D83, 084019 (2011), arXiv:1011.5120 [hep-th], doi:10.1103/PhysRevD.83.084019.

66. M. Berasaluce-González, L. E. Ibáñez, P. Soler and A. M. Uranga, JHEP 1112, 113 (2011), arXiv: 1106.4169 [hep-th], doi:10.1007/JHEP12(2011)113.

67. L. Ibáñez, A. Schellekens and A. Uranga, Nucl.Phys. B865, 509 (2012), arXiv:1205.5364 [hep-th], doi:10.1016/j.nuclphysb.2012.08.008.

68. G. Honecker and W. Staessens, J. Phys. Conf. Ser. 631, 012080 (2015), arXiv:1502.00985 [hep-th], doi:10.1088/1742-6596/631/1/012080.

69. R. Peccei and H. R. Quinn, Phys.Rev.Lett. 38, 1440 (1977), doi:10.1103/ PhysRevLett.38.1440.

70. G. Honecker, Fortsch. Phys. 64, 380 (2016), arXiv:1510.08443 [hep-th], doi:10. 1002/prop.201500064.

71. S. A. Abel, S. Sarkar and P. L. White, Nucl. Phys. B454, 663 (1995), arXiv:hep-ph/9506359 [hep-ph], doi:10.1016/0550-3213(95)00483-9.

72. M. Cvetič, R. Donagi, D. Klevers, H. Piragua and M. Poretschkin, Nucl. Phys. B898, 
736 (2015), arXiv:1502.06953 [hep-th], doi:10.1016/j.nuclphysb.2015.07.011.

73. R. Blumenhagen, M. Cvetič, F. Marchesano and G. Shiu, JHEP 0503, 050 (2005), arXiv:hep-th/0502095 [hep-th], doi:10.1088/1126-6708/2005/03/050.

74. T. W. Grimm and J. Louis, Nucl.Phys. B718, 153 (2005), arXiv:hep-th/0412277 [hep-th], doi:10.1016/j.nuclphysb.2005.04.007.

75. T. W

Grimm and D. V. Lopes, Nucl.Phys. B855, 639 (2012), arXiv:1104.2328 [hep-th], doi: 10.1016/j.nuclphysb.2011.10.019.

76. M. Kerstan and T. Weigand, JHEP 1106, 105 (2011), arXiv:1104.2329 [hep-th], doi:10.1007/JHEP06(2011)105.

77. M. B. Green and J. H. Schwarz, Phys.Lett. B149, 117 (1984), doi:10.1016/ 0370-2693(84)91565-X.

78. R. Blumenhagen, G. Honecker and T. Weigand, JHEP 0508, 009 (2005), arXiv:hep-th/0507041 [hep-th], doi:10.1088/1126-6708/2005/08/009.

79. R. Blumenhagen, G. Honecker and T. Weigand, Non-Abelian brane worlds: The Open string story, in 2nd Southeastern European Workshop on Challenges Beyond the Standard Model (BW2005) Vrnjacka Banja, Serbia, Nis, Serbia, May 19-23, 2005, (2005). arXiv:hep-th/0510050 [hep-th].

80. D. Lüst, P. Mayr, R. Richter and S. Stieberger, Nucl.Phys. B696, 205 (2004), arXiv:hep-th/0404134 [hep-th], doi:10.1016/j.nuclphysb.2004.06.052.

81. I. Antoniadis, C. Bachas and E. Dudas, Nucl. Phys. B560, 93 (1999), arXiv:hep-th/9906039 [hep-th], doi:10.1016/S0550-3213(99)00452-6.

82. C. G. Callan, Jr., C. Lovelace, C. R. Nappi and S. A. Yost, Nucl. Phys. B288, 525 (1987), doi:10.1016/0550-3213(87)90227-6.

83. C. Bachas and M. Porrati, Phys. Lett. B296, 77 (1992), arXiv:hep-th/9209032 [hep-th], doi:10.1016/0370-2693(92)90806-F.

84. N. Akerblom, R. Blumenhagen, D. Lüst and M. Schmidt-Sommerfeld, JHEP 0708, 044 (2007), arXiv:0705.2366 [hep-th], doi:10.1088/1126-6708/2007/08/044.

85. A. Hebecker, A. K. Knochel and T. Weigand, JHEP 06, 093 (2012), arXiv:1204.2551 [hep-th], doi:10.1007/JHEP06(2012)093.

86. D. Cremades, L. E. Ibáñez and F. Marchesano, JHEP 07, 038 (2003), arXiv:hep-th/0302105 [hep-th], doi:10.1088/1126-6708/2003/07/038.

87. M. Cvetič

and I. Papadimitriou, Phys.Rev. D68, 046001 (2003), arXiv:hep-th/0303083 [hep-th], doi:10.1103/PhysRevD.68.046001,10.1103/PhysRevD.70.029903.

88. S. Abel and A. Owen, Nucl.Phys. B663, 197 (2003), arXiv:hep-th/0303124 [hep-th], doi:10.1016/S0550-3213(03)00370-5.

89. S. Abel and A. Owen, Nucl.Phys. B682, 183 (2004), arXiv:hep-th/0310257 [hep-th], doi:10.1016/j.nuclphysb.2003.11.032.

90. M. Cvetič, G. Shiu and A. M. Uranga, Phys.Rev.Lett. 87, 201801 (2001), arXiv:hep-th/0107143 [hep-th], doi:10.1103/PhysRevLett.87.201801.

91. M. Cvetič, G. Shiu and A. M. Uranga, Nucl.Phys. B615, 3 (2001), arXiv:hep-th/0107166 [hep-th], doi:10.1016/S0550-3213(01)00427-8.

92. G. Honecker, Nucl.Phys. B666, 175 (2003), arXiv:hep-th/0303015 [hep-th], doi: 10.1016/S0550-3213(03)00540-6.

93. G. Honecker, String phenomenology. Proceedings, 2nd International Conference, Durham, UK, July 29-August 4, 2003 , 191 (2003), arXiv:hep-th/0309158 [hep-th].

94. G. Honecker, Mod.Phys.Lett. A19, 1863 (2004), arXiv:hep-th/0407181 [hep-th], doi:10.1142/S0217732304015087. 
95. S. Förste and C. Liyanage, JHEP 03, 110 (2015), arXiv:1412.3645 [hep-th], doi: 10.1007/JHEP03(2015)110.

96. I. Pesando, Nucl. Phys. B910, 618 (2016), arXiv:1512.07920 [hep-th], doi:10. 1016/j.nuclphysb.2016.06.013.

97. R. Blumenhagen, L. Görlich and T. Ott, JHEP 0301, 021 (2003), arXiv:hep-th/0211059 [hep-th].

98. D.

Bailin

and A. Love, Nucl.Phys. B755, 79 (2006), arXiv:hep-th/0603172 [hep-th], doi: 10.1016/j.nuclphysb.2006.07.025,10.1016/j.nuclphysb.2007.08.005.

99. D. Bailin and A. Love, Phys.Lett. B651, 324 (2007), arXiv:0705.0646 [hep-th], doi:10.1016/j.physletb.2007.06.042,10.1016/j.physletb.2007.11.014.

100. D. Bailin and A. Love, Nucl.Phys. B809, 64 (2009), arXiv:0801.3385 [hep-th], doi:10.1016/j.nuclphysb.2008.09.036.

101. G. Honecker and J. Vanhoof, JHEP 1204, 085 (2012), arXiv:1201.3604 [hep-th], doi:10.1007/JHEP04(2012)085.

102. G. Honecker and J. Vanhoof, Fortsch.Phys. 60, 1050 (2012), arXiv:1201.5872 [hep-th], doi:10.1002/prop.201200016.

103. D. Bailin and A. Love, JHEP 1401, 009 (2014), arXiv:1310.8215 [hep-th], doi: 10.1007/JHEP01(2014)009.

104. G. Honecker and W. Staessens, PoS Corfu2012, 107 (2013), arXiv:1303.6845 [hep-th].

105. E. Cremmer, S. Ferrara, L. Girardello and A. Van Proeyen, Nucl.Phys. B212, 413 (1983), doi:10.1016/0550-3213(83)90679-X.

106. L. J. Dixon, V. Kaplunovsky and J. Louis, Nucl.Phys. B329, 27 (1990), doi:10.1016/ 0550-3213(90)90057-K.

107. M.

M. Haack and J. U. Kang, JHEP 1211, 091 (2012), arXiv:1112.5156 [hep-th], doi:10.1007/JHEP11(2012)091.

108. M. Berg, M. Haack, J. U. Kang and S. Sjörs, JHEP 12, 077 (2014), arXiv:1407.0027 [hep-th], doi:10.1007/JHEP12(2014)077.

109. S. Förste and I. Zavala, JHEP 0807, 086 (2008), arXiv:0806.2328 [hep-th], doi: 10.1088/1126-6708/2008/07/086.

110. R. Blumenhagen, M. Cvetič, S. Kachru and T. Weigand, Ann.Rev.Nucl.Part.Sci. 59, 269 (2009), arXiv:0902.3251 [hep-th], doi:10.1146/annurev.nucl.010909.083113.

111. M. Cvetič and J. Halverson, TASI Lectures: Particle Physics from Perturbative and Non-perturbative Effects in D-braneworlds, in Proceedings, Theoretical Advanced Study Institute in Elementary Particle Physics (TASI 2010). String Theory and Its Applications: From meV to the Planck Scale: Boulder, Colorado, USA, June 1-25, 2010, (2011), pp. 245-292. arXiv:1101.2907 [hep-th].

112. F. Marchesano, D. Regalado and G. Zoccarato, JHEP 11, 097 (2014), arXiv:1410.0209 [hep-th], doi:10.1007/JHEP11(2014)097.

113. D. Bailin and A. Love, Nucl.Phys. B854, 700 (2012), arXiv:1104.3522 [hep-th], doi:10.1016/j.nuclphysb.2011.09.018.

114. M. Blaszczyk, G. Honecker and I. Koltermann, JHEP 1407, 124 (2014), arXiv: 1403.2394 [hep-th], doi:10.1007/JHEP07(2014)124.

115. M. Blaszczyk, G. Honecker and I. Koltermann, JHEP 11, 019 (2015), arXiv:1507.07568 [hep-th], doi:10.1007/JHEP11(2015)019.

116. I. Koltermann, M. Blaszczyk and G. Honecker, Fortsch. Phys. 64, 412 (2016), arXiv:1511.03549 [hep-th], doi:10.1002/prop.201500073.

117. I. Koltermann, Geometry and supersymmetry in type II string theory, PhD thesis, 
Mainz U., Institut für Physik (2016-05).

118. C. Vafa and E. Witten, J.Geom.Phys. 15, 189 (1995), arXiv:hep-th/9409188 [hep-th], doi:10.1016/0393-0440(94)00048-9.

119. G. Honecker, I. Koltermann and W. Staessens (2016), MITP/16-103.

120. G. Villadoro and F. Zwirner, JHEP 03, 087 (2006), arXiv:hep-th/0602120 [hep-th], doi:10.1088/1126-6708/2006/03/087.

121. R. Blumenhagen, S. Moster and E. Plauschinn, JHEP 01, 058 (2008), arXiv:0711.3389 [hep-th], doi:10.1088/1126-6708/2008/01/058.

122. R. Blumenhagen, G. Honecker and T. Weigand, JHEP 0510, 086 (2005), arXiv:hep-th/0510049 [hep-th], doi:10.1088/1126-6708/2005/10/086.

123. G. Honecker, Nucl. Phys. B748, 126 (2006), arXiv:hep-th/0602101 [hep-th], doi: 10.1016/j.nuclphysb.2006.04.027.

124. R. Blumenhagen, G. Honecker and T. Weigand, JHEP 0506, 020 (2005), arXiv:hep-th/0504232 [hep-th], doi:10.1088/1126-6708/2005/06/020.

125. R. Blumenhagen, S. Moster and T. Weigand, Nucl.Phys. B751, 186 (2006), arXiv:hep-th/0603015 [hep-th], doi:10.1016/j.nuclphysb.2006.06.005.

126. R. Blumenhagen, S. Moster, R. Reinbacher and T. Weigand, JHEP 05, 041 (2007), arXiv:hep-th/0612039 [hep-th], doi:10.1088/1126-6708/2007/05/041.

127. R. Blumenhagen, B. Jurke, T. Rahn and H. Roschy, J. Math. Phys. 51, 103525 (2010), arXiv:1003.5217 [hep-th], doi:10.1063/1.3501132,10.1063/1.3523343.

128. E. I. Buchbinder, A. Constantin and A. Lukas, JHEP 03, 025 (2014), arXiv:1311.1941 [hep-th], doi:10.1007/JHEP03(2014)025.

129. S. Groot Nibbelink, O. Loukas, F. Ruehle and P. K. S. Vaudrevange, Phys. Rev. D92, 046002 (2015), arXiv: 1506.00879 [hep-th], doi:10.1103/PhysRevD.92.046002.

130. R. Slansky, Phys. Rept. 79, 1 (1981), doi:10.1016/0370-1573(81)90092-2.

131. D. R. Morrison and C. Vafa, Nucl. Phys. B473, 74 (1996), arXiv:hep-th/9602114 [hep-th], doi:10.1016/0550-3213(96)00242-8.

132. D. R. Morrison and C. Vafa, Nucl. Phys. B476, 437 (1996), arXiv:hep-th/9603161 [hep-th], doi:10.1016/0550-3213(96)00369-0.

133. T. Weigand, Class.Quant.Grav. 27, 214004 (2010), arXiv:1009.3497 [hep-th], doi: 10.1088/0264-9381/27/21/214004.

134. R. Donagi and M. Wijnholt, Adv.Theor.Math.Phys. 15, 1237 (2011), arXiv:0802.2969 [hep-th], doi:10.4310/ATMP.2011.v15.n5.a2.

135. C. Beasley, J. J. Heckman and C. Vafa, JHEP 0901, 058 (2009), arXiv:0802.3391 [hep-th], doi: 10.1088/1126-6708/2009/01/058.

136. J. J. Heckman, D. R. Morrison and C. Vafa, JHEP 05, 028 (2014), arXiv:1312.5746 [hep-th], doi:10.1007/JHEP06(2015)017,10.1007/JHEP05(2014) 028, [Erratum: JHEP06,017(2015)].

137. J. J. Heckman, D. R. Morrison, T. Rudelius and C. Vafa, Fortsch. Phys. 63, 468 (2015), arXiv:1502.05405 [hep-th], doi:10.1002/prop.201500024.

138. L. B. Anderson, J. Gray, D. Grayson, Y.-H. He and A. Lukas, Commun. Math. Phys. 297, 95 (2010), arXiv:0904.2186 [hep-th], doi:10.1007/s00220-010-1033-8.

139. L. B. Anderson, J. Gray and B. Ovrut, JHEP 05, 086 (2010), arXiv:1001.2317 [hep-th], doi:10.1007/JHEP05(2010)086.

140. V. Braun, M. Kreuzer, B. A. Ovrut and E. Scheidegger, Phys. Lett. B649, 334 (2007), arXiv:hep-th/0703134 [hep-th], doi:10.1016/j.physletb.2007.03.066.

141. V. Braun, M. Kreuzer, B. A. Ovrut and E. Scheidegger, JHEP 10, 022 (2007), arXiv:hep-th/0703182 [HEP-TH], doi:10.1088/1126-6708/2007/10/022.

142. V. Braun, M. Kreuzer, B. A. Ovrut and E. Scheidegger, JHEP 10, 023 (2007), 
arXiv:0704.0449 [hep-th], doi:10.1088/1126-6708/2007/10/023.

143. G. Honecker and M. Trapletti, JHEP 01, 051 (2007), arXiv:hep-th/0612030 [hep-th], doi:10.1088/1126-6708/2007/01/051.

144. G. Honecker (2007), arXiv:0709.2037 [hep-th].

145. M. Blaszczyk, S. Nibbelink Groot, F. Ruehle, M. Trapletti and P. K. Vaudrevange, JHEP 1009, 065 (2010), arXiv:1007.0203 [hep-th], doi:10.1007/JHEP09(2010) 065 .

146. S. Groot Nibbelink, Fortsch. Phys. 59, 454 (2011), arXiv:1012.3350 [hep-th], doi: 10.1002/prop.201100002.

147. M. Blaszczyk, S. Groot Nibbelink and F. Ruehle, JHEP 05, 053 (2012), arXiv:1111.5852 [hep-th], doi:10.1007/JHEP05(2012)053.

148. H. P. Nilles, S. Ramos-Sanchez, P. K. Vaudrevange and A. Wingerter, Comput.Phys.Commun. 183, 1363 (2012), arXiv:1110.5229 [hep-th], doi:10.1016/j. cpc.2012.01.026.

149. T. Kobayashi, S. L. Parameswaran, S. Ramos-Sanchez and I. Zavala, JHEP 05, 008 (2012), arXiv:1107.2137 [hep-th], doi:10.1007/JHEP12(2012)049,10.1007/ JHEP05(2012)008, [Erratum: JHEP12,049(2012)].

150. O. Lebedev, H. P. Nilles, S. Raby, S. Ramos-Sanchez, M. Ratz et al., Phys.Lett. B645, 88 (2007), arXiv:hep-th/0611095 [hep-th], doi:10.1016/j.physletb.2006.12.012.

151. O. Lebedev, H. P. Nilles, S. Ramos-Sanchez, M. Ratz and P. K. Vaudrevange, Phys.Lett. B668, 331 (2008), arXiv:0807.4384 [hep-th], doi:10.1016/j.physletb. 2008.08.054.

152. R. Donagi and K. Wendland, J. Geom. Phys. 59, 942 (2009), arXiv:0809.0330 [hep-th], doi:10.1016/j.geomphys.2009.04.004.

153. P. Athanasopoulos, A. E. Faraggi, S. Groot Nibbelink and V. M. Mehta, JHEP 04, 038 (2016), arXiv: 1602.03082 [hep-th], doi:10.1007/JHEP04(2016)038.

154. M. Fischer, M. Ratz, J. Torrado and P. K. S. Vaudrevange, JHEP 01, 084 (2013), arXiv:1209.3906 [hep-th], doi:10.1007/JHEP01(2013)084.

155. P. Anastasopoulos, T. Dijkstra, E. Kiritsis and A. Schellekens, Nucl.Phys. B759, 83 (2006), arXiv:hep-th/0605226 [hep-th], doi:10.1016/j.nuclphysb.2006.10.013.

156. R. Peccei and H. R. Quinn, Phys.Rev. D16, 1791 (1977), doi:10.1103/PhysRevD.16. 1791.

157. P. Svrcek and E. Witten, JHEP 0606, 051 (2006), arXiv:hep-th/0605206 [hep-th], doi:10.1088/1126-6708/2006/06/051.

158. D. Berenstein and E. Perkins, Phys.Rev. D86, 026005 (2012), arXiv:1202.2073 [hep-th], doi:10.1103/PhysRevD.86.026005.

159. G. Honecker and W. Staessens, Fortsch. Phys. 62, 115 (2014), arXiv:1312.4517 [hep-th], doi:10.1002/prop.201300036.

160. A. R. Zhitnitsky, Sov. J. Nucl. Phys. 31, 260 (1980), [Yad. Fiz.31,497(1980)].

161. M. Dine, W. Fischler and M. Srednicki, Phys.Lett. B104, 199 (1981), doi:10.1016/ 0370-2693(81)90590-6.

162. R. Blumenhagen, D. Lüst and S. Stieberger, JHEP 07, 036 (2003), arXiv:hep-th/0305146 [hep-th], doi:10.1088/1126-6708/2003/07/036.

163. L. A. Anchordoqui, I. Antoniadis, H. Goldberg, X. Huang, D. Lüst et al., Phys.Rev. D85, 086003 (2012), arXiv: 1107.4309 [hep-ph], doi:10.1103/PhysRevD.85.086003.

164. M. Cvetič, J. Halverson and P. Langacker, JHEP 1111, 058 (2011), arXiv:1108.5187 [hep-ph], doi:10.1007/JHEP11(2011)058.

165. R. F. Lebed and V. E. Mayes, Phys. Rev. D84, 075016 (2011), arXiv:1106.4347 [hep-ph], doi:10.1103/PhysRevD.84.075016.

166. M. R. Buckley, D. Hooper, J. Kopp and E. Neil, Phys. Rev. D83, 115013 (2011), 
arXiv:1103.6035 [hep-ph], doi:10.1103/PhysRevD.83.115013.

167. L. A. Anchordoqui, I. Antoniadis, H. Goldberg, X. Huang, D. Lüst et al., Phys.Rev. D86, 066004 (2012), arXiv:1206.2537 [hep-ph], doi:10.1103/PhysRevD.86.066004.

168. D. Berenstein, Ann. Rev. Nucl. Part. Sci. 64, 197 (2014), arXiv:1401.4491 [hep-th], doi:10.1146/annurev-nucl-102313-025342.

169. G. Ballesteros, J. Redondo, A. Ringwald and C. Tamarit (2016), arXiv:1608.05414 [hep-ph].

170. S. Abel, M. Goodsell, J. Jaeckel, V. Khoze and A. Ringwald, JHEP 0807, 124 (2008), arXiv:0803.1449 [hep-ph], doi:10.1088/1126-6708/2008/07/124. 